\title{
Experimental evaluation of cylinder vortex shedding noise reduction using porous material
}

\author{
Thomas F. Geyer ${ }^{1}$
}

Received: 2 February 2020 / Revised: 1 May 2020 / Accepted: 6 May 2020 / Published online: 13 June 2020

(c) The Author(s) 2020

\begin{abstract}
The tonal noise generation of a circular cylinder in a uniform flow is an important source of aerodynamic noise. It can be found at parts of the landing gear of airplanes, at pantographs of trains, at antennas and basically all other protruding parts of vehicles. This noise is due to the periodic shedding of vortices along the cylinder span. One method to reduce this noise is the use of flow permeable covers around the cylinders. In the present study, measurements were performed in an aeroacoustic wind tunnel on a large set of porous covered cylinders. In addition to varying the porous material, which is characterized by its air flow resistivity and its porosity, the thickness of the porous layer was varied as well. The measurements were performed at Reynolds numbers between 14,000 and 103,000 using microphones located in the acoustic far field. It was found that the porous covers lead to a notable narrowing of the vortex shedding tonal peak in the sound pressure level spectra, an effect that increases with increasing porosity and thickness and decreasing air flow resistivity of the porous layer. Based on the large set of experimental data, basic trends were derived for the estimation of the vortex shedding Strouhal number and the reduction in the energy in the vortex shedding peak using the method of linear regression. Constant temperature anemometry measurements in the wake of selected cylinders basically showed a similar narrowing of the vortex shedding peak in the spectra of the turbulent velocity fluctuations. In addition, the measurement of wake profiles showed a reduction in the mean velocity and the turbulence in the wake as well as a widening of the wake region, while an analysis of the spanwise coherence revealed that the cause of the overall noise reduction is not a breakup of spanwise turbulent structures. Rather, the results imply that viscous damping of turbulent flow pressure amplitudes by the porous material strongly contributes to the noise reduction.
\end{abstract}

\section{Graphic abstract}

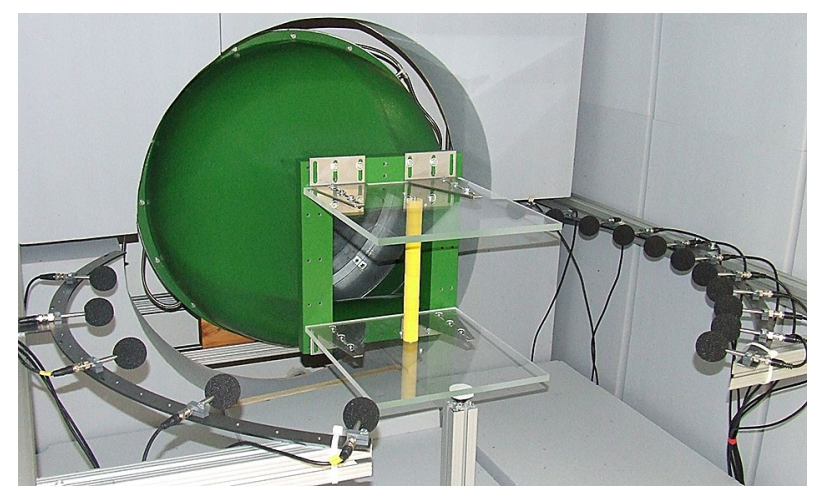

Thomas F. Geyer

thomas.geyer@b-tu.de

1 Brandenburg University of Technology Cottbus Senftenberg, 03046 Cottbus, Germany

\section{Introduction}

When a circular cylinder is placed in a crossflow, so-called Kármán vortices are shed periodically, which results in strong tonal noise known as the aeolian tone (Strouhal 1878). Many methods are known for the reduction of this 
noise (Rashidi et al. 2016), with the use of porous materials being one that receives increasing attention. Thus, several studies exist on the use of flow permeable materials for the reduction of aerodynamic noise from cylinders. This group of studies can be divided into those in which a total suppression of the vortex shedding tonal noise was observed (such as Nishimura et al. 1999; Sueki et al. 2010; Nishimura and Goto 2010; Aguiar et al. 2016; Geyer 2020) and those in which the vortex shedding noise was reduced, but not completely suppressed (for example the work of Akishita and Yahathugoda 2005; Sueki et al. 2010; Liu et al. 2012; Arcondoulis et al. 2019; Geyer and Sarradj 2016).

Of course, attention has also been paid to the aerodynamic effect of such porous covered cylinders. Hence, a very important question is how the use of porous cylinder covers alters the flow around the cylinders. Basically, several effects have been described in previous studies. ${ }^{1}$ One major observation that has been reported often is that porous cylinder covers regularize or stabilize the wake flow. This was found both in numerical studies (Bruneau and Mortazavi 2008; Liu et al. 2012; Naito and Fukagata 2012) and in experimental investigations (Sueki et al. 2010; Aguiar et al. 2016; Showkat Ali et al. 2016; Klausmann and Ruck 2017; Xia et al. 2018; Liu et al. 2019; Geyer 2020). Furthermore, it has also been observed that porous cylinder coatings lead to a delay of the vortex roll-up, and hence a downstream shift of the region where the vortex street formation occurs (Liu et al. 2012; Showkat Ali et al. 2016; Yuan et al. 2016; Klausmann and Ruck 2017; Xia et al. 2018). As a consequence, many researchers reported a broadening or widening of the wake (Nishimura et al. 1999; Suzuki et al. 2009; Nishimura and Goto 2010; Ashtiani Abdi et al. 2014; Aguiar et al. 2016; Yuan et al. 2016; Xia et al. 2018; Liu et al. 2019; Sadeghipour et al. 2020), while, conversely, a reduction of this width has also been reported (Klausmann and Ruck 2017). The latter, however, was for cases where the cylinder was not fully covered by porous material, it was only attached to the leeward side. An increase in the length of the wake has also been reported (Suzuki et al. 2009; Sueki et al. 2010; Gozmen et al. 2013; Rashidi et al. 2013; Ashtiani Abdi et al. 2014; Xia et al. 2018; Sadeghipour et al. 2020). Other effects of the porous materials are a reduction of the mean velocity (Aguiar et al. 2016; Yuan et al. 2016; Xia et al. 2018; Yuan et al. 2019) and the turbulence intensity or the turbulent kinetic energy in the wake (Suzuki et al. 2009; Nishimura and Goto 2010; Sueki et al. 2010; Gozmen et al. 2013; Aguiar et al. 2016; Showkat Ali et al. 2016; Liu et al. 2019; Sadeghipour et al. 2020).

\footnotetext{
$\overline{1}$ This includes only studies on solid cylinders covered by a porous layer, not fully porous cylinders or hollow porous cylinders.
}

Regarding the effect of porous cylinder covers on the drag coefficient, opposite observations can be found: Some studies showed that such covers can reduce the drag (Bhattacharyya and Singh 2011; Liu et al. 2012; Klausmann and Ruck 2017), while others observed an increase in drag (Zhao and Cheng 2010; Naito and Fukagata 2012; Yuan et al. 2016). Showkat Ali et al. (2016) observed a drag reduction in the subcritical regime, but an increase in drag in the critical regime due to porous cylinder covers.

In addition, the fact that the use of porous layers for microphone windscreens also leads to a reduction of wind noise, which depends on the properties of the porous materials (Xu et al. 2011; Zhao et al. 2017), shall also be mentioned here for completeness.

Most of the studies on porous covered cylinders, however, were performed for a limited number of porous materials. Furthermore, the thickness of the porous covers is often kept constant as well. In one of the experimental studies, performed by Geyer and Sarradj (2016), the aerodynamic noise generated by two-dimensional circular cylinders covered with different porous materials of the same thickness was examined. It was found that the porous materials investigated did not lead to a complete suppression of the vortex shedding tonal noise, but rather to a narrowing of the tonal peak. This is in agreement with the numerical findings from Liu et al. (2012) and with experimental measurements of the base pressure spectrum from Klausmann and Ruck (2017).

The aim of the present study is to continue the previous investigation (Geyer and Sarradj 2016), but to additionally vary the thickness of the porous layer. Furthermore, a large number of porous materials were newly acquired to complement the materials from Geyer and Sarradj (2016). Based on the large resulting data set, the method of linear regression was used to derive basic trends for the influence of the material parameters on the noise reduction that can be achieved. In addition to the acoustic measurements, constant temperature anemometry (CTA) measurements were performed in the wake of selected cylinders to enable further insight into the effect of the porous covers on the adjacent flow field.

\section{Materials and methods}

\subsection{Wind tunnel}

All experiments took place in the small aeroacoustic wind tunnel facility at the Brandenburg University of Technology (Sarradj et al. 2009), which is an open jet wind tunnel driven by a radial fan with a shaft power of $18.5 \mathrm{~kW}$ and which can be equipped with nozzles of different shapes and dimensions. For the current study, a nozzle with a rectangular exit area of $0.23 \mathrm{~m} \times 0.28 \mathrm{~m}$ was used, allowing for a maximum flow velocity of about $60 \mathrm{~m} / \mathrm{s}$. The nozzle was equipped 
on two sides with side walls made of acrylic glass, which had dimensions of $0.4 \mathrm{~m}$ in the streamwise direction and $0.38 \mathrm{~m}$ in the horizontal direction. The cylinder models were attached to these side walls on both ends, with a distance of $0.15 \mathrm{~m}$ between the cylinder axis and the nozzle exit. At this position, the turbulent boundary layer thickness is below $10 \mathrm{~mm}$ (Moreau et al. 2018). Figure 1 shows a photograph of the setup, while Fig. 2 shows a corresponding schematic.

The flow velocity $U_{0}$ in the facility is set by adjusting the pressure in the wind tunnel settling chamber, which is then used to calculate the velocity at the nozzle exit using Bernoulli's principle.

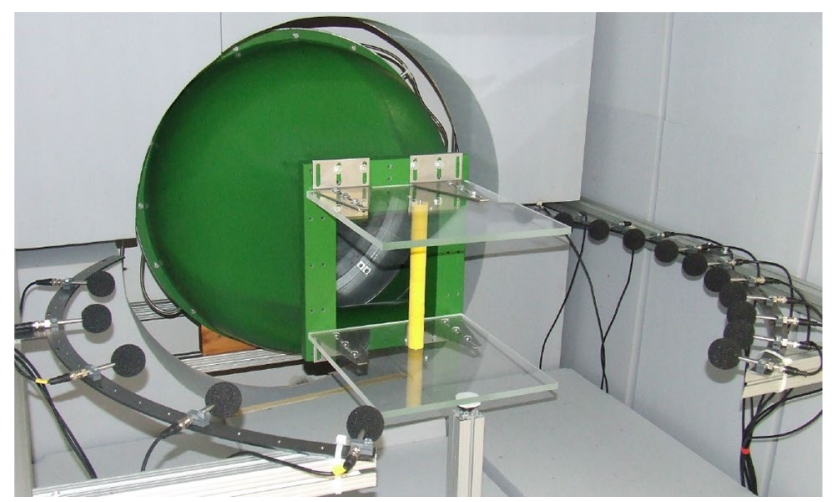

Fig. 1 Photograph of the setup used for the acoustic measurements

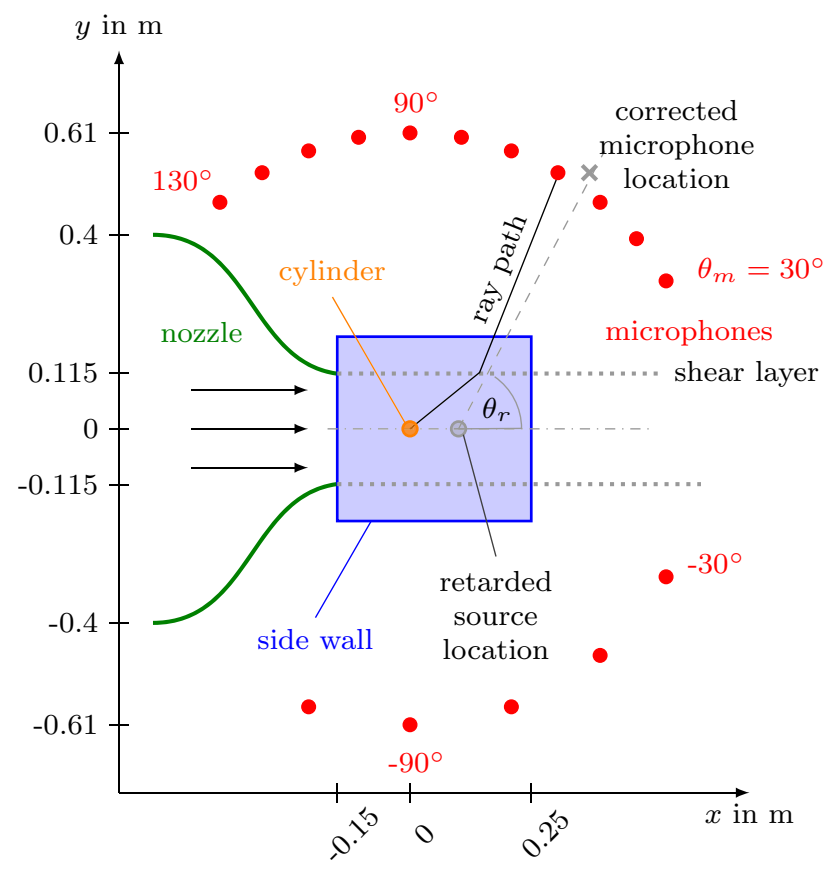

Fig. 2 Schematic of the setup used for the acoustic measurements (top view; including the ray path from the cylinder to the microphone at $\theta_{m}=+60^{\circ}$ and corresponding corrected microphone location according to Amiet 1978)
During acoustic measurements, the test section is surrounded by a cabin which has absorbing walls on five sides (only the side opposite to the wind tunnel nozzle is open), providing an anechoic environment for frequencies above approximately $125 \mathrm{~Hz}$.

\subsection{Porous covered cylinders}

The porous covered cylinders consisted of a non-porous core cylinder made of polyurethane, on which cylindrical slices of porous material were threaded. Regarding the dimensions of the cylinder models, several requirements had to be taken into account: Firstly, according to the literature (Porteous et al. 2014), no Kármán vortex shedding may occur for cylinders with aspect ratios (which is the ratio of cylinder length to cylinder diameter) below 7. Secondly, the cylinder diameter should not be too large in order to keep the blockage of the wind tunnel as small as possible. However, thirdly, due to manufacturing reasons, the diameter should also not be too small. Finally, cylinders were designed for which the outer diameter $D$ was kept constant at $D=30 \mathrm{~mm}$, while the inner diameter $d$ of the core cylinder was varied between a minimum value of $6 \mathrm{~mm}$ and a maximum value of $22 \mathrm{~mm}$. Thus, the thickness of the porous layer $t=(D-d) / 2$ ranged from $4 \mathrm{~mm}$ to $12 \mathrm{~mm}$. The length $L$ of the cylinders was $0.28 \mathrm{~m}$, resulting in an aspect ratio $L / D$ of 9.3 .

In total, 17 different open-porous materials were used as covers. They are mainly characterized by their air flow resistivity

$r=\frac{\Delta p \cdot A}{q \cdot h}$,

which is a measure for the resistance of a porous material against a fluid flow through the material, and is, for a given fluid, inversely proportional to the viscous permeability $k_{v}$. The air flow resistivity was measured according to ISO 9053 (ISO 9053 1993). In Eq. (1), $q$ is a static, unidirectional volume flow through a cylindrical sample of the porous material with thickness $h$ and cross-sectional area $A$ and $\Delta p$ is the static pressure difference across the sample. The aim of the present study was to use a variety of different porous materials with a range of air flow resistivities as large as possible. In addition to the air flow resistivity, which is assumed to have the biggest influence on a potential noise reduction (Geyer et al. 2010), the volume porosity $\sigma$ is also believed to be important, as materials with very low porosities were found to not lead to a sufficient reduction in aerodynamic noise (Geyer 2011; Geyer and Sarradj 2019). The porosity was estimated using the total density $\rho_{t}$ of the porous material (including the pores), which was either taken from the datasheet or measured, and the density of the skeletal material $\rho_{s}$ (without the pores) according to 
Table 1 Overview of the cylinders used in the study

\begin{tabular}{|c|c|c|c|c|c|}
\hline Material & Description & $\begin{array}{l}\text { Outer diam- } \\
\text { eter } D(\mathrm{~mm})\end{array}$ & Inner diameter $d(\mathrm{~mm})$ & $\begin{array}{l}\text { Air flow resistiv- } \\
\text { ity } r(\mathrm{~Pa} \mathrm{~s} / \mathrm{m})\end{array}$ & Porosity $\sigma(-)$ \\
\hline Reference & Non-porous & 30 & - & $\infty$ & 0 \\
\hline Reference, tripped & Non-porous & 30 & - & $\infty$ & 0 \\
\hline Damtec Black Uni & Polyurethane/cork granulate & 30 & 10 & $1,474,300$ & $0.4 \ldots 0.6$ \\
\hline Damtec Standard & Rubber/cork granulate & 30 & 10 & 594,200 & $0.18 \ldots 0.32$ \\
\hline Oasis Rainbow Foam & Synthetic resin foam & 30 & 10 & 416,200 & $0.97 \ldots 0.98$ \\
\hline Regufoam V 300 FR & Polyurethane elastomer foam & 30 & $10 ; 20$ & 381,500 & $0.69 \ldots 0.75$ \\
\hline Regufoam Vibration 190 plus & Polyurethane foam & 30 & 10 & 314,800 & $0.81 \ldots 0.85$ \\
\hline Getzner CM GR 0525 & Polyurethane foam & 30 & $6 ; 10 ; 15 ; 20 ; 22$ & 141,300 & $0.71 \ldots 0.77$ \\
\hline ArmaFoam Sound & Elastomer foam & 30 & 10 & 112,100 & $0.85 \ldots 0.9$ \\
\hline Damtec SBM K 20 & Rubber granulate & 30 & $6 ; 10 ; 15 ; 20 ; 22$ & 86,100 & $0.29 \ldots 0.32$ \\
\hline Regufoam Vibration 150 plus & Polyurethane foam & 30 & $10 ; 22$ & 64,500 & $0.85 \ldots 0.88$ \\
\hline Conmetall Rubber Mat & Rubber granulate & 30 & $6 ; 10 ; 22$ & 53,200 & $0.14 \ldots 0.18$ \\
\hline Regupol Vibration 800 & Bound rubber fibers & 30 & $10 ; 20$ & 49,600 & $0.19 \ldots 0.23$ \\
\hline Damtec estra & Rubber granulate & 30 & $6 ; 10 ; 15 ; 20 ; 22$ & 12,900 & $0.18 \ldots 0.29$ \\
\hline Basotect & Melamine resin foam & 30 & $10 ; 13 ; 20$ & 9800 & $>0.99$ \\
\hline Damtec Black Rubber & Rubber granulate & 30 & $6 ; 10 ; 15 ; 20$ & 9400 & $0.23 \ldots 0.27$ \\
\hline Regupol Vibration 550 & Bound rubber fibers & 30 & $6 ; 10 ; 22$ & 4400 & $0.25 \ldots 0.28$ \\
\hline Packing Foam & Polyurethane foam & 30 & 10 & 4100 & $>0.99$ \\
\hline Panacell 90 ppi & Polyurethane foam & 30 & 10 & 4000 & $>0.99$ \\
\hline
\end{tabular}

$\sigma=1-\frac{\rho_{t}}{\rho_{s}}$

It has to be noted that the porosity values calculated for the present porous covers should be understood as approximate values only, especially for the granulate materials. This is due to the fact that these materials are often conglomerates containing more than one basic material (such as rubber and cork) plus some kind of binding agent, which makes it hard to obtain exact values for the densities of the skeletal material.

The materials were cut into shape using water jet cutting, since other technologies that generate heat (such as laser cutting or milling) are very likely to destroy or close the pores on the surface. The disadvantage of water jet cutting, however, is that it did not yield tolerable results for materials with very low air flow resistivities and high porosities, because those are usually very soft. Hence, the porous materials of the current study have rather medium and high air flow resistivities.

From each material, at least one cylinder cover was cut, using an inner diameter of $10 \mathrm{~mm}$. In order to also evaluate the influence of the thickness of the porous layer, cylinder covers with differing inner diameters $d$ were cut from several of the materials. For the purpose of comparison, a non-porous reference cylinder with the same (outer) diameter of $30 \mathrm{~mm}$ was additionally used. In addition, a second reference cylinder was used for the acoustic measurements, which had thin tripping tape with a width of $1 \mathrm{~mm}$ and a height of $0.2 \mathrm{~mm}$ applied at an angle of $\pm 50^{\circ}$ to the flow (following the work of Igarashi 1986). Thus, in the end, measurements were performed on a total of 43 single cylinder models. Table 1 gives an overview of all cylinders used, while Fig. 3 exemplarily shows a photograph of a subset of the cylinder models.

\subsection{Acoustic measurement setup}

The measurements were done using sixteen $1 / 4$ th inch free field microphones (type MI-17, with a nominal sensitivity of $50 \mathrm{mV} / \mathrm{Pa}$ and a typical frequency response of $\pm 0.5 \mathrm{~dB}$ in a frequency range from $20 \mathrm{~Hz}$ to $4 \mathrm{kHz}$ ) arranged on two

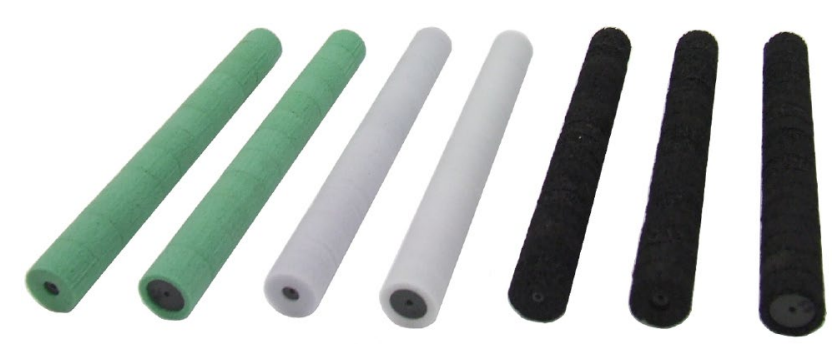

Fig. 3 Photograph of some of the cylinders (left: two cylinders covered with Regufoam Vibration 150 plus, center: two cylinders covered with Basotect, right: three cylinders covered with Regupol Vibration 550) 
arcs positioned on opposite sides of the test section, with a radial distance of $0.61 \mathrm{~m}$. As is visible in Fig. 2, one arc contained eleven microphones, ranging from an azimuthal angle of $+30^{\circ}$ to $+130^{\circ}$ in $10^{\circ}$ steps. The other arc contained five microphones, ranging from $-30^{\circ}$ to $-110^{\circ}$ in $20^{\circ}$ steps.

The data were recorded with a sampling frequency of $51.2 \mathrm{kHz}$ and a duration of $90 \mathrm{~s}$ using a National Instruments 24 Bit multichannel measurement system. In postprocessing, the time data were converted to the frequency domain using a fast Fourier transformation (FFT) on Hanning-windowed blocks of 65,536 samples with an overlap of $75 \%$. This leads to spectra of the sound pressure with a small frequency step size of only $0.8 \mathrm{~Hz}$. Finally, the data were converted to sound pressure levels $L_{p}$ with a reference value of $20 \mu \mathrm{Pa}$.

\subsection{Constant temperature anemometry setup}

In addition to the acoustic measurements, CTA measurements were performed on a subset of the porous covered cylinders, with the aim to help understand the effect of the porous covers on the flow in the vicinity of the cylinders. Three different kinds of investigation were performed: In the first one, profiles of the mean velocity and the turbulence intensity were measured in the wake of the cylinders, using a Dantec P11-type single wire probe. These wake profiles were obtained at two downstream locations: the first one outer diameter $D$ downstream from the cylinder axis (labeled the "near wake") and the second 10.5 outer diameters $D$ downstream from the cylinder axis (labeled the "far wake"). Thereby, the distance between neighboring measurement locations in the lateral direction was varied. It was $0.5 \mathrm{~mm}$ in the region downstream from the cylinder, with an extent of $1 D$ to each side from the axis, and was increased to $1 \mathrm{~mm}$ further to the side. The probe was positioned using a three-dimensional traverse system with a minimum step size of $0.1 \mathrm{~mm}$. The data were recorded with a sampling frequency of $25.6 \mathrm{kHz}$ and a measurement duration of $12 \mathrm{~s}$ using a 24 Bit National Instruments digital signal acquisition module. In post-processing, the first $2 \mathrm{~s}$ of each data set were omitted to account for possible vibrations of the hotwire probe after each step of the traverse system. From the remaining data, both the mean velocity $U$ and the turbulence intensity $T u=\tilde{u} / U_{0}$ were calculated, $\tilde{u}$ being the root-meansquare value of the measured velocity fluctuations.

In a second investigation, power spectral densities of the turbulent velocity fluctuations in the wake of the cylinders were measured. This was again done using a single wire probe, which, in agreement with (Bearman 1969; Kamps et al. 2017), was positioned one outer diameter $D$ downstream of the cylinder and one half outer diameter off center. The data were recorded with the same sampling frequency of $25.6 \mathrm{kHz}$, but an increased measurement duration of
$80 \mathrm{~s}$. The time data were then transferred into the frequency domain using an FFT. This was done blockwise on blocks of 32,768 samples with an overlap of $50 \%$, resulting in the same frequency spacing as used for the acoustic spectra.

In the third investigation, the spanwise coherence $\gamma^{2}(f)$ between two identical Dantec single wire probes was measured. The first probe was located at a fixed position, one outer diameter $D$ downstream of the cylinder, aligned with its axis, with a distance of one $D$ from the side wall. The second probe was traversed in the spanwise direction away from the first probe, with a step size of $1 / 12 D$. The coherence function can then be calculated from the resulting auto spectral densities $G_{1,1}(f)$ and $G_{2,2}(f)$ and the cross spectral density $G_{1,2}(f)$ from the probe signals according to (Bendat and Piersol (2010))

$\gamma^{2}(f)=\frac{\left|G_{1,2}(f)\right|^{2}}{G_{1,1}(f) \cdot G_{2,2}(f)}$.

Basically, the coherence is a measure for the relation between two signals. It is a frequency-dependent parameter that can take values between zero and one. If it is equal to zero, the two signals are completely unrelated, and if it approaches one, the signals are related. The latter could occur for example when both signals are the output of a linear system that are caused by the same input signal. The spanwise coherence can provide information on the size of coherent structures in the wake of the cylinders and thus help to understand how the porous consistency changes the flow field and, subsequently, the generation of aerodynamic noise. These measurements were done with the same sampling frequency of $25.6 \mathrm{kHz}$, but a shorter measurement duration of $10 \mathrm{~s}$ per measurement point due to the large number of points.

\section{Results and discussion}

\subsection{Acoustic measurements}

For each cylinder, measurements were conducted at 14 flow speeds between $7 \mathrm{~m} / \mathrm{s}$ and $52 \mathrm{~m} / \mathrm{s}$, leading to Reynolds numbers $R e_{D}$ (based on outer cylinder diameter $D$ ) between 14,000 and 103,000 . Thus, the flow regime is the subcritical one (Fey et al. 1998; Hutcheson and Brooks 2012).

\subsubsection{Overview}

In a first step, the influence of the porous material and the influence of the material thickness on the resulting sound pressure level spectra will be shown individually at a single flow speed, based on measurements taken with the microphone at an angle of $90^{\circ}$ (see Fig. 2). As shown in Fig. 4, the 


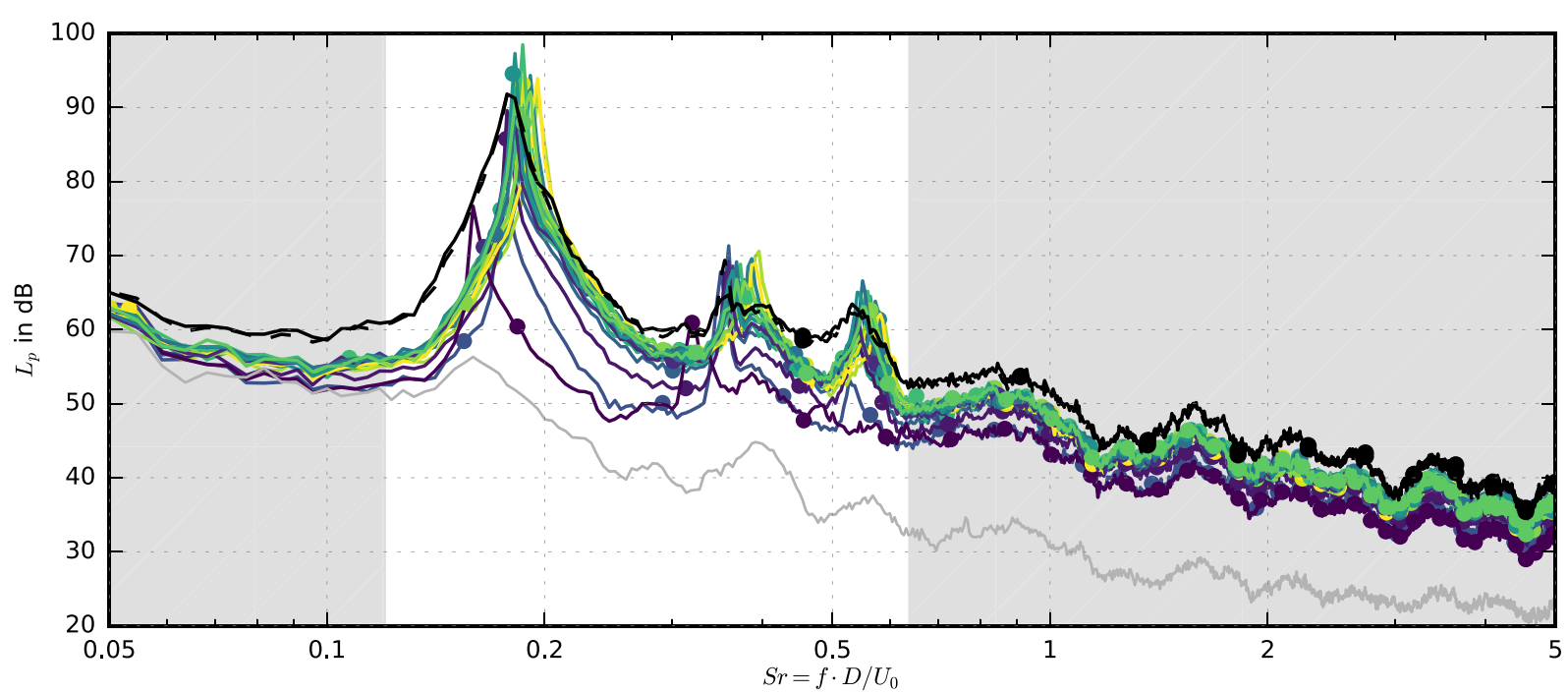

Fig. 4 Sound pressure level spectra obtained for cylinders covered with different materials at $R e_{D}=99,000$ with the microphone positioned at $90^{\circ}$ to the flow, $d=10 \mathrm{~mm}(\square$ non-porous, $\boldsymbol{\square}$ nonporous tripped, porous: $r=1,474,300 \mathrm{~Pa} \mathrm{~s} / \mathrm{m}^{2}, \quad 594,200 \mathrm{~Pa} \mathrm{~s} /$ $\mathrm{m}^{2},-416,200 \mathrm{~Pa} \mathrm{~s} / \mathrm{m}^{2},-381,500 \mathrm{~Pa} \mathrm{~s} / \mathrm{m}^{2},-314,800 \mathrm{~Pa} \mathrm{~s} /$ $\mathrm{m}^{2}, 141,300 \mathrm{~Pa} \mathrm{~s} / \mathrm{m}^{2}, 112,100 \mathrm{~Pa} \mathrm{~s} / \mathrm{m}^{2}, \quad 86,100 \mathrm{~Pa} \mathrm{~s} / \mathrm{m}^{2}$,

porous material has a notable influence on both the broadband noise and the tonal noise, which was already observed in a past study (Geyer and Sarradj 2016). In general, the spectra show three distinct tonal peaks. The first, which is due to classical Kármán vortex shedding, appears at a Strouhal number $\mathrm{Sr}$ of 0.18 (corresponding to a frequency around $300 \mathrm{~Hz}$ ), while the first two harmonics of this tone appear as considerably smaller peaks at twice and three times this frequency. Regarding the broadband noise, it is visible that materials with low air flow resistivities lead to a greater noise reduction than materials with higher air flow resistivities. At frequencies above the third peak, it can be seen that the sound pressure level differences are in the order of up to $10 \mathrm{~dB}$. Regarding the tonal noise, it is visible that the porous covers lead to a narrowing of the vortex shedding peak, but not to its complete suppression. This was already observed in a previous study (Geyer and Sarradj 2016) and agrees well with the findings from Liu et al. (2012), who show numerically obtained far field sound pressure level spectra, and Klausmann and Ruck (2017), who show measured surface pressure spectra. It is also visible that the frequency of the vortex shedding peak is different for the different materials, an effect that will be investigated more closely in Sect. 3.1.3. In addition, Fig. 4 shows that the sound pressure level spectrum obtained for the reference cylinder with tripping tape is nearly identical to that obtained for the untripped reference cylinder.

The effect of the thickness of the porous layer on the noise generation is then shown in Fig. 5, which is done
64,500 Pa s $/ \mathrm{m}^{2}$, 53,200 Pa s/m² 49,600 Pa s/m², $12,900 \mathrm{~Pa} \mathrm{~s} / \mathrm{m}^{2}, \quad 9800 \mathrm{~Pa} \mathrm{~s} / \mathrm{m}^{2}, \quad 9400 \mathrm{~Pa} \mathrm{~s} / \mathrm{m}^{2}, 4400 \mathrm{~Pa} \mathrm{~s} /$ $\mathrm{m}^{2}, 4100 \mathrm{~Pa} \mathrm{~s} / \mathrm{m}^{2}, 4000 \mathrm{~Pa} \mathrm{~s} / \mathrm{m}^{2}$ ), gray line: background noise level, measured without a cylinder, but with the side plates installed; gray hatched area: broadband noise

exemplarily for two porous materials. The first one (Fig. 5a), Basotect, has a low air flow resistivity of only $9800 \mathrm{~Pa} \mathrm{~s} / \mathrm{m}^{2}$ and a high porosity $\sigma>0.99$, while the second, Damtec SBM K 20 (Fig. 5b), has a considerably higher air flow resistivity of $86,100 \mathrm{~Pa} \mathrm{~s} / \mathrm{m}^{2}$ and a lower porosity between 0.29 and 0.32 . Basically, it can be seen that an increase in thickness, and hence a decrease in inner diameter $d$, leads to a further increase in broadband noise reduction as well as a decrease in amplitude and a further narrowing of the vortex shedding peak. However, these effects are clearly visible for the material with the low air flow resistivity, while they are much weaker for the materials with high air flow resistivity. For the porous covers with even higher air flow resistivities, such as Regufoam V 300 FR $\left(r=381,500 \mathrm{~Pa} \mathrm{~s} / \mathrm{m}^{2}\right)$ or Getzner CM GR 0525 $\left(r=141,300 \mathrm{~Pa} \mathrm{~s} / \mathrm{m}^{2}\right)$, the differences are almost negligible (not shown here for brevity).

It can also be concluded from Fig. 4 and, especially, Fig. 5, that there is no hint of any vortex shedding from the core cylinder, which would lead to additional tonal peaks at a range of different Strouhal numbers in the sound pressure level spectra. Instead, the main vortex shedding peak always appears in the same range of frequencies as that of the non-porous reference cylinders, revealing that the outer diameter $D$ is the essential dimension regarding the vortex shedding. This effect, which is in agreement with the previous study (Geyer and Sarradj 2016) as well as with results from other researchers (Yuan et al. 2019), 


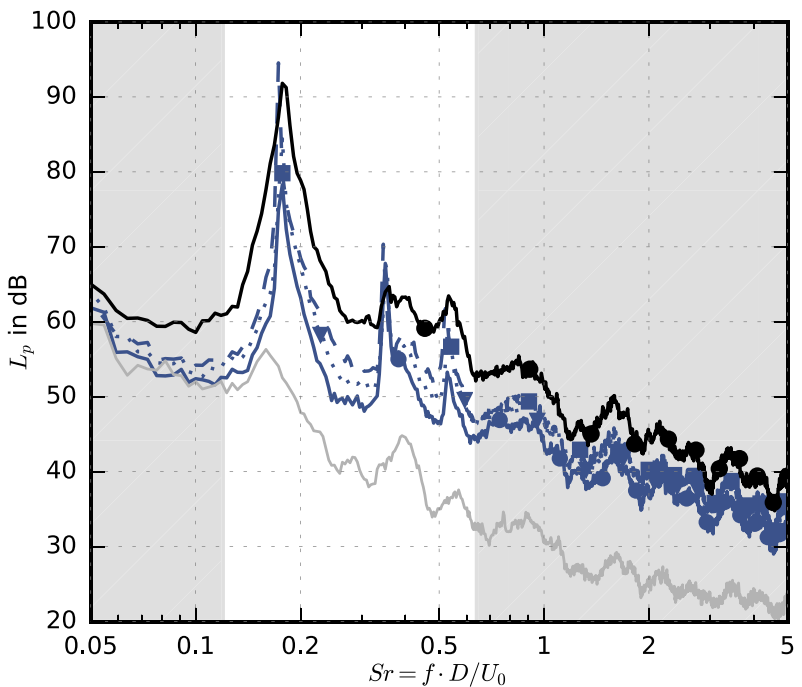

(a) Basotect, $r=9,800 \mathrm{~Pa} \mathrm{~s} / \mathrm{m}^{2}$ ( non-porous, porous: - $d=10 \mathrm{~mm}, \boldsymbol{\nabla} d=13 \mathrm{~mm}, \boldsymbol{\square} d=20 \mathrm{~mm}$ ), gray line: background noise level, measured without a cylinder, but with the side plates installed; gray hatched area: broadband noise.

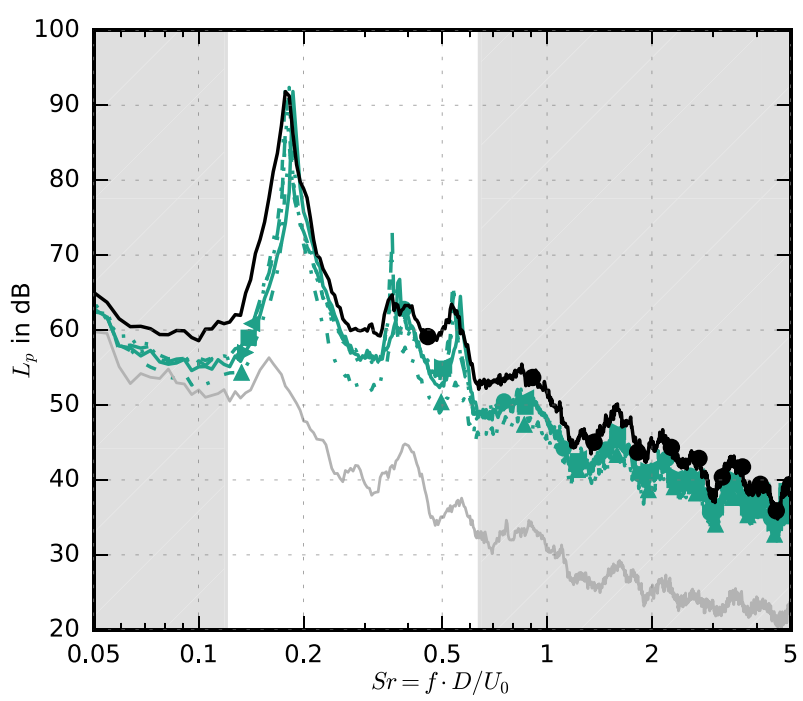

(b) Damtec SBM K 20, $r=86,100 \mathrm{~Pa} \mathrm{~s} / \mathrm{m}^{2}$ ( non-porous, porous: $\Delta d=6 \mathrm{~mm}, \bullet d=10 \mathrm{~mm}, \nabla d=15 \mathrm{~mm}$, $d=20 \mathrm{~mm}, \varangle d=22 \mathrm{~mm}$ ), gray line: background noise level, measured without a cylinder, but with the side plates installed; gray hatched area: broadband noise.

Fig. 5 Sound pressure level spectra obtained for cylinders covered with materials of different thickness at $R e_{D}=99,000$ with the microphone positioned at $90^{\circ}$ to the flow

will be further analyzed in Sect. 3.2 using results from hot wire measurements.

In the following sections, the acoustic effect of the porous covers on both the broadband noise and on the tonal noise due to periodic vortex shedding will be examined in more detail.

\subsubsection{Effect on broadband noise}

In order to analyze the effect of the porous covers on the broadband noise generation, an overall sound pressure level for the broadband noise was calculated according to

$O S P L=10 \cdot \log _{10}\left(\sum 10^{L_{p} / 10 \mathrm{~dB}}\right) \mathrm{dB}$.

Thereby, the integration included the sound pressure levels at all frequencies for which the Strouhal number was either smaller than 0.12 or greater than 0.65 , which corresponds to the gray hatched areas in Figs. 4 and 5. Thus, the tonal part of the sound pressure level spectrum, which contains the vortex shedding tone and its first two harmonics, was omitted from the integration. This was done for the data recorded with the microphone at an angle of $90^{\circ}$.

Figure 6 shows the resulting broadband overall sound pressure levels as a function of Reynolds number $R e_{D}$ and Mach number $M=U_{0} / c$ (with $c$ being the speed of sound) for all cylinders of the present study. Overall, it is visible that the broadband noise level approximately increases with the sixth power of the Mach number. Regarding the influence of the porous materials, it can be observed that materials with low air flow resistivities result in the lowest broadband noise levels, with noise reductions in the order of $10 \mathrm{~dB}$. This confirms the basic findings from Fig. 4. Still, all porous covered cylinders of the present study lead to a reduction of broadband noise compared to the reference cylinder, even those with very high air flow resistivities. While the broadband noise reduction is very small at low Reynolds numbers, it is more pronounced at Reynolds numbers above approximately 50,000 . There, the difference between the reference cylinder and the porous covered cylinders is at least $2 \mathrm{~dB}$ (for materials with high air flow resistivities) and more (for materials with low air flow resistivities).

The present results agree well with those from Liu et al. (2012) and with those from Klausmann and Ruck (2017), who also report a dampening of the broadband fluctuations of the pressure around a cylinder modified with porous material on the leeward side.

\subsubsection{Effect on tonal noise}

In what follows, the effects of the porous covers on the tonal peak due to classical Kármán vortex shedding will be examined, using the data measured by the microphone at $90^{\circ}$ only. Due to the large amount of data, this is done in a more statistical way by pointing out basic trends and trying to derive simple mathematical relationships for these dependencies.

Firstly, the effect of the porous covers on the peak Strouhal number $S r_{\text {peak }}$ is shown in Fig. 7 as a function of Reynolds number and Mach number. For the bare reference cylinder and 


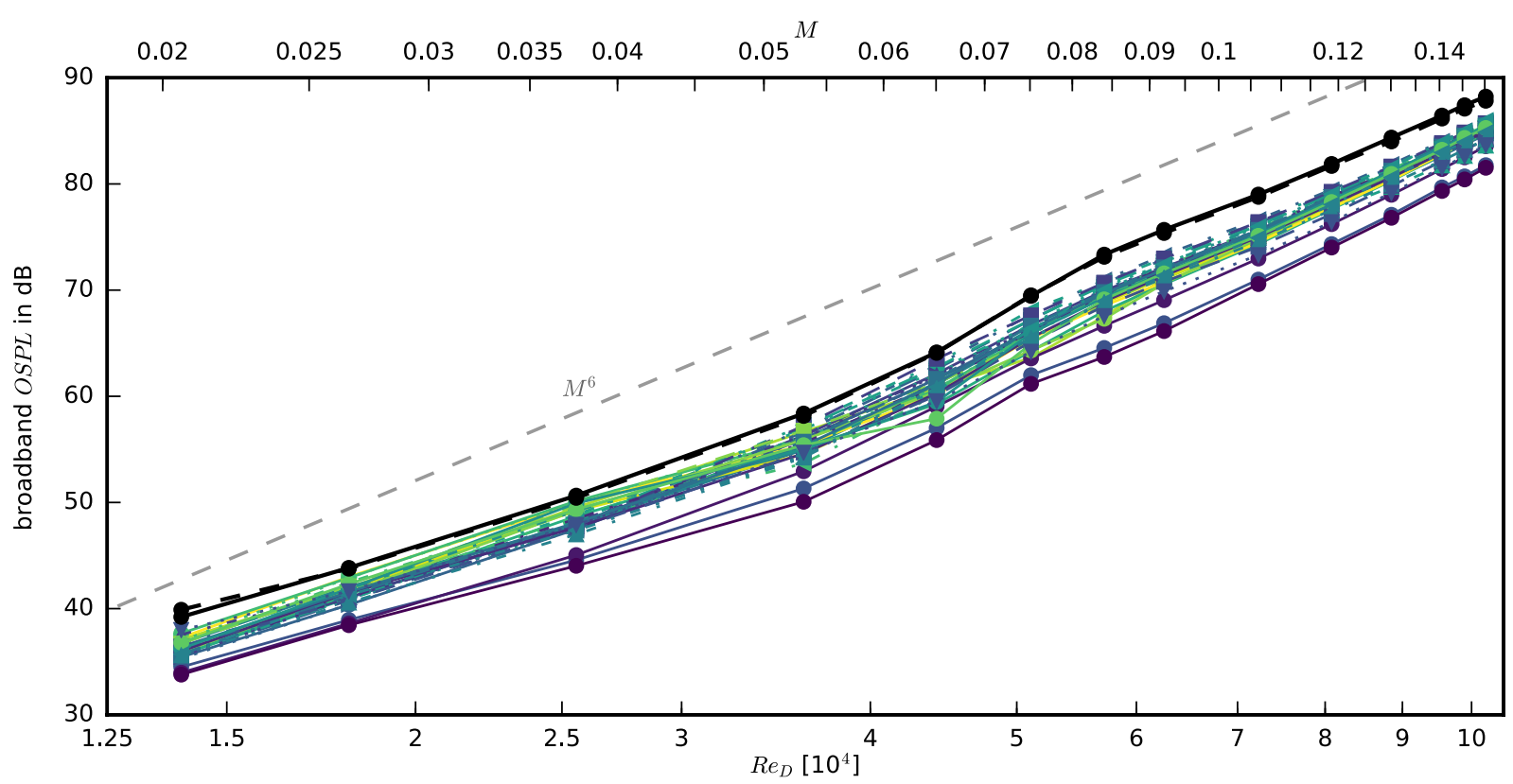

Fig. 6 Broadband overall sound pressure levels, calculated according to Eq. (4), using data obtained by the microphone at $90^{\circ}$ for all cylinders from Table 1, materials are color-coded ( nonporous, - non-porous tripped, porous: $r=1,474,300 \mathrm{~Pa}$ s/ $\mathrm{m}^{2}$, 594,200 Pa s/m² $416,200 \mathrm{~Pa} \mathrm{~s} / \mathrm{m}^{2}$, 381,500 Pa s/ $\mathrm{m}^{2}, 314,800 \mathrm{~Pa} \mathrm{~s} / \mathrm{m}^{2}, 141,300 \mathrm{~Pa} \mathrm{~s} / \mathrm{m}^{2}, 112,100 \mathrm{~Pa} \mathrm{~s} /$ $\mathrm{m}^{2},-86,100 \mathrm{~Pa} \mathrm{~s} / \mathrm{m}^{2},-64,500 \mathrm{~Pa} \mathrm{~s} / \mathrm{m}^{2}, \quad 53,200 \mathrm{~Pa} \mathrm{~s} / \mathrm{m}^{2}$, 49,600 Pa s/m², 12,900 Pa s/m², $9800 \mathrm{~Pa} \mathrm{~s} / \mathrm{m}^{2}, \quad 9400 \mathrm{~Pa} \mathrm{~s} /$ $\mathrm{m}^{2}, 4400 \mathrm{~Pa} \mathrm{~s} / \mathrm{m}^{2}, \quad 4100 \mathrm{~Pa} \mathrm{~s} / \mathrm{m}^{2}, 4000 \mathrm{~Pa} \mathrm{~s} / \mathrm{m}^{2}$ ), thicknesses of the porous layer are given by different markers and line styles $(\Delta d=6 \mathrm{~mm}, \bullet d=10 \mathrm{~mm}, \boldsymbol{\nabla} d=13 \mathrm{~mm}, \boldsymbol{\nabla} d=15 \mathrm{~mm}, \boldsymbol{}$ $d=20 \mathrm{~mm}, \varangle d=22 \mathrm{~mm}$ )

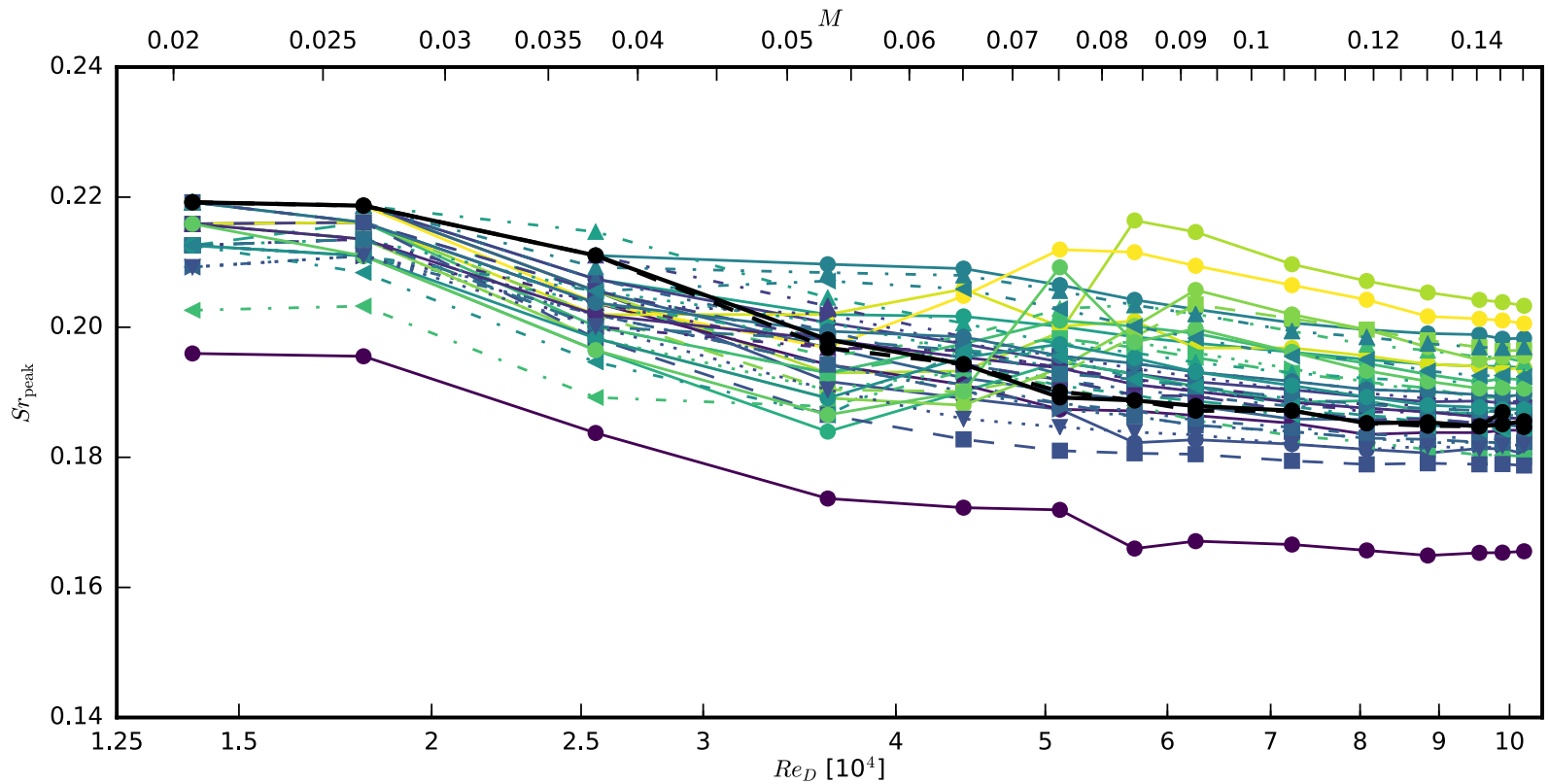

Fig. 7 Strouhal number of the Kármán vortex shedding peak obtained by the microphone at $90^{\circ}$ for all cylinders from Table 1 , materials are color-coded ( non-porous, $\boldsymbol{\square}$ non-porous tripped, porous: $r=1,474,300 \mathrm{~Pa} \mathrm{~s} / \mathrm{m}^{2}, \quad 594,200 \mathrm{~Pa} \mathrm{~s} / \mathrm{m}^{2}, \quad 416,200 \mathrm{~Pa} \mathrm{~s} /$ $\mathrm{m}^{2},-381,500 \mathrm{~Pa} \mathrm{~s} / \mathrm{m}^{2},-314,800 \mathrm{~Pa} \mathrm{~s} / \mathrm{m}^{2}$, $141,300 \mathrm{~Pa} \mathrm{~s} /$ $\mathrm{m}^{2}, 112,100 \mathrm{~Pa} \mathrm{~s} / \mathrm{m}^{2}, \quad 86,100 \mathrm{~Pa} \mathrm{~s} / \mathrm{m}^{2}, \quad 64,500 \mathrm{~Pa} \mathrm{~s} / \mathrm{m}^{2}$,

the tripped reference cylinder, the Strouhal number slightly decreases from 0.22 at the lowest Reynolds number to about
53,200 Pa s/m² - 49,600 Pa s/m², 12,900 Pa s/m², $9800 \mathrm{~Pa} \mathrm{~s} / \mathrm{m}^{2}, \quad 9400 \mathrm{~Pa} \mathrm{~s} / \mathrm{m}^{2}, 4400 \mathrm{~Pa} \mathrm{~s} / \mathrm{m}^{2}, 4100 \mathrm{~Pa} \mathrm{~s} / \mathrm{m}^{2}$, - $4000 \mathrm{~Pa} \mathrm{~s} / \mathrm{m}^{2}$ ), thicknesses of the porous layer are given by different markers and line styles $(\Lambda d=6 \mathrm{~mm}, \boldsymbol{\bullet} d=10 \mathrm{~mm}, \boldsymbol{\nabla} d=13 \mathrm{~mm}$, $\triangleright d=15 \mathrm{~mm}, \boldsymbol{=} d=20 \mathrm{~mm}, \varangle d=22 \mathrm{~mm})$

0.18 at the highest Reynolds number. These values agree well with the literature (Blake et al. 1986; Fey et al. 1998). 
The slight decrease in the Strouhal number with increasing Reynolds number has also been observed in the previous study (Geyer and Sarradj 2016) as well as in experiments by Hutcheson and Brooks on cylinders of a similar diameter (Hutcheson and Brooks 2012). Regarding the porous covers, a clear trend is visible: At low Reynolds numbers $R e_{D}<25,000$, the Strouhal numbers obtained for the porous covered cylinders are below that of the reference cylinders. With increasing $R e_{D}$, the Strouhal numbers of the porous covered cylinders decrease as well. However, for most materials, this decrease occurs with a lower slope than for the non-porous reference cylinders. Thus, at high Reynolds numbers, cylinders covered with materials with medium and high air flow resistivities lead to a higher Strouhal number than that of the non-porous reference cylinder. Only cylinders covered with materials with very low air flow resistivities lead to Strouhal numbers below that of the reference cylinders. This result agrees well with that from the previous study by the author (Geyer and Sarradj 2016).

In a next step, the method of linear regression (Willis et al. 1997; Schmidt and Lipson 2009; Sarradj and Geyer 2014) was used to derive basic trends for the dependencies of the peak Strouhal number of the vortex shedding tone shown in Fig. 7. By using this method, it is possible to formulate mathematical equations of varying accuracy and complexity only based on a given dataset. For the present study, the aim is to find simple formulas that allow for a better understanding of the influence of the different input parameters rather than to identify a very complex equation that might have a very high accuracy. At the same time, the models should, if possible, comply with theoretical dependencies known for dipole noise sources. In the present case, the method was applied to a total number of 588 data tuples, from which $50 \%$ were used as training data and $50 \%$ as validation data.

It is reasonable to assume that the peak Strouhal number depends on the properties of the porous materials (the air flow resistivity $r$ and the porosity $\sigma$ ), the thickness of the porous covers $t$ and the Reynolds number or the Mach number. Since the outer diameter was kept constant and the temperature did not vary much during experiments, both the Reynolds number and the Mach number more or less depend only on the flow speed and thus will lead to similar results. However, a dependency of the Strouhal number on the Reynolds number according to

$S r=S r^{*}+\frac{m}{\sqrt{R e}}$

can be expected according to Fey et al. (1998), where $\mathrm{Sr}^{*}$ and $m$ are constants. In the subcritical flow regime, these constants were found to be $\mathrm{Sr}^{*}=0.1776$ and $m=2.2023$. Hence, it was decided to use the Reynolds number instead of the Mach number for the Strouhal number model, and a dependency on $1 / \sqrt{\operatorname{Re}}$ was demanded.
Since a physically meaningful mathematical model requires dimensionless parameters, the term

$\zeta=\frac{\rho c}{r D}$

was defined, which can be understood as a normalized permeability of the porous materials, with $\rho$ being the density of air. The air flow resistivity $r$ appears in the denominator, which was chosen on purpose in order to obtain a value of $\zeta=0$ for the non-porous reference cylinder (instead of a value of infinity, which is much harder to handle mathematically). Table 2 lists the possible input parameters and their ranges for the linear regression analysis. The thickness of the porous covers was non-dimensionalized using the outer diameter $D$ of the cylinders.

One of the resulting mathematical expressions, which considers all parameters of the porous covers and at the same time fulfills the requirement regarding a preferably high level of simplicity, is given by

$$
\begin{aligned}
S r= & 0.1668+0.0222 \frac{t}{D}-0.0076 \zeta \sigma+28.4872 \zeta R e^{-1} \\
& +5.2953 R e^{-\frac{1}{2}} .
\end{aligned}
$$

This formulation has a goodness of fit of 0.75 and a mean absolute error of 0.004 . Despite this relatively large error, it nevertheless allows for several significant conclusions: Firstly, the normalized permeability according to Eq. (6) and the porosity appear together in one term, showing that both parameters together have an influence on the Strouhal number. This term has a negative sign, meaning that both a high air flow resistivity and a low porosity will lead to an increase in the peak Strouhal number. This agrees well with the spectra shown in Fig. 4 and the results shown in Fig. 7. In addition, the normalized permeability $\zeta$ appears in a second term that also contains the Reynolds number, which accounts for the different slopes observed in Fig. 7 for the different materials. Secondly, the Strouhal number increases with increasing thickness of the porous layer, which is visible (although not as clearly as the influence of the air flow resistivity and the porosity) in Fig. 5. In addition, as mentioned above, a theoretical dependence on $1 / \sqrt{R e}$ was maintained. It has to be noted, of course, that Eq. (7) was derived based on the

Table 2 Input parameters of the porous cylinder covers for the linear regression analysis

\begin{tabular}{ll}
\hline Parameter & Range \\
\hline Normalized permeability $\zeta($ Eq. (6)) & $0.009 \ldots 3.464$ \\
Porosity $\sigma$ & $0.16 \ldots 0.99$ \\
Normalized thickness of porous cover $t / D$ & $0.133 \ldots 0.4$ \\
Mach number $M$ & $0.02 \ldots 0.15$ \\
Reynolds number $R e_{D}$ & $13,990 \ldots 102,200$ \\
\hline
\end{tabular}




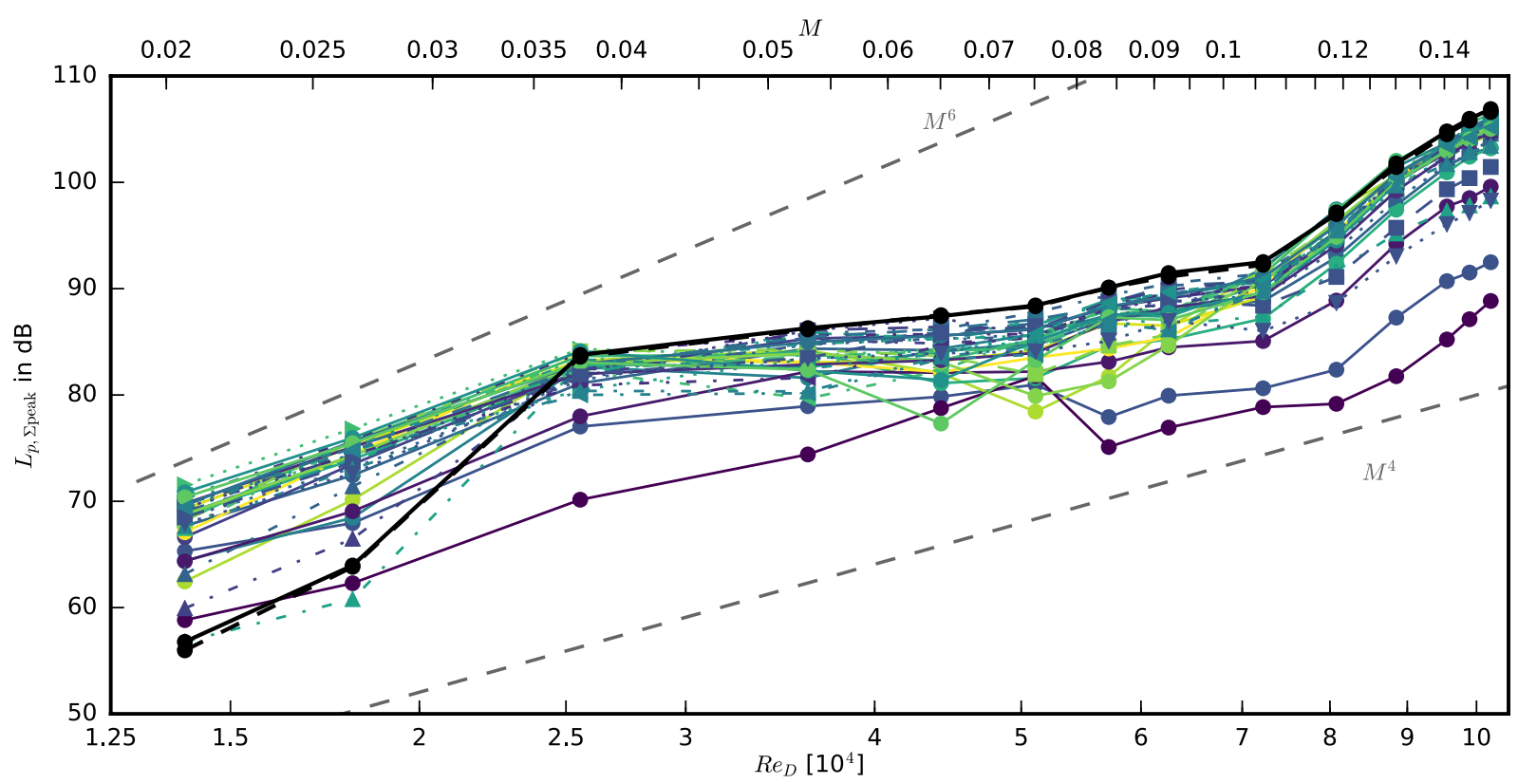

Fig. 8 Energy in the Kármán vortex shedding peak obtained by the microphone at $90^{\circ}$ for all cylinders from Table 1 , materials are color-coded ( non-porous, - non-porous tripped, porous: $r=1,474,300 \mathrm{~Pa} \mathrm{~s} / \mathrm{m}^{2}, \quad 594,200 \mathrm{~Pa} \mathrm{~s} / \mathrm{m}^{2}, \quad 416,200 \mathrm{~Pa} \mathrm{~s} /$ $\mathrm{m}^{2}, \quad 381,500 \mathrm{~Pa} \mathrm{~s} / \mathrm{m}^{2}, \quad 314,800 \mathrm{~Pa} \mathrm{~s} / \mathrm{m}^{2}, \quad 141,300 \mathrm{~Pa} \mathrm{~s} /$ $\mathrm{m}^{2},-112,100 \mathrm{~Pa} \mathrm{~s} / \mathrm{m}^{2}, \quad 86,100 \mathrm{~Pa} \mathrm{~s} / \mathrm{m}^{2}, \quad 64,500 \mathrm{~Pa} \mathrm{~s} / \mathrm{m}^{2}$,

parameter ranges specified in Table 2, and hence it may not be valid for parameters outside these ranges.

The significance of the contribution of the single parameters to the formulation given in Eq. (7) can be quantified using the sensitivity metric, which specifies the relative impact of a variable $x_{i}(i=1,2, \ldots n)$ on a target function $g=f\left(x_{1}, x_{2}, \ldots x_{n}\right)$ according to

$\overline{\left|\frac{\partial g}{\partial x_{i}}\right|} \cdot \frac{\sigma\left(x_{i}\right)}{\sigma(g)}$

where $\frac{\partial g}{\partial x_{i}}$ is the partial derivative of $g$ with respect to $x_{i}$ and $\sigma()$ is the standard deviation of the respective variable. The sensitivities for the different input variables for the model according to Eq. (7) are 0.97 for the Reynolds number, 0.32 for the parameter $\zeta, 0.25$ for the normalized thickness $t / D$ and 0.20 for the porosity $\sigma$.

As a second measure for the analysis of the tonal noise generated by the cylinders, the energy of the tonal peak $L_{p, \Sigma \text { peak }}$ is shown in Fig. 8 for all cylinders of the present study as a function of the Reynolds number $R e_{D}$. As in Geyer and Sarradj (2016), it is defined as the integral of the energy in the Kármán vortex shedding peak between the two points where the level is $10 \mathrm{~dB}$ below the maximum.

In general, it can be expected that the peak energy is a function of the Mach number. Furthermore, for a theoretical dipole noise source a dependence on $M^{6}$ should be
53,200 Pa s/m², 49,600 Pa s/m², 12,900 Pa s/m², $9800 \mathrm{~Pa} \mathrm{~s} / \mathrm{m}^{2}, \quad 9400 \mathrm{~Pa} \mathrm{~s} / \mathrm{m}^{2}, 4400 \mathrm{~Pa} \mathrm{~s} / \mathrm{m}^{2}, 4100 \mathrm{~Pa} \mathrm{~s} / \mathrm{m}^{2}$, - $4000 \mathrm{~Pa} \mathrm{~s} / \mathrm{m}^{2}$ ), thicknesses of the porous layer are given by different markers and line styles $(\boldsymbol{\Delta} d=6 \mathrm{~mm}, \bullet d=10 \mathrm{~mm}, \boldsymbol{\nabla} d=13 \mathrm{~mm}$, $\triangleright d=15 \mathrm{~mm}, \mathbf{\square} d=20 \mathrm{~mm}, \Delta d=22 \mathrm{~mm})$

visible (see, for example, Keefe 1962). In experimental studies on finite cylinders, however, the overall velocity scaling may differ from the theoretical scaling. For example, the peak levels shown by Hutcheson and Brooks (2012) for cylinders of similar diameter as in the present study are scaled with $M^{6}$, but the resulting curves still show a slight decrease with increasing Mach number. In the present study, the peak energies are found to scale with the fourth to sixth power of the Mach number, which agrees with the findings from the previous study (Geyer and Sarradj 2016). Interestingly, the results seem to show some kind of transition in the scaling behavior: For Reynolds numbers below approximately 25,000 and above 60,000, an overall scaling close to the theoretical $M^{6}$ dependency is visible (although the exponent seems to be slightly lower in the lower range). In the region between those two Reynolds numbers, the scaling exponent is even lower. The reason for this change is not clear yet, but it has also been observed in the previous study (Geyer and Sarradj 2016) using a different experimental setup. One possibility is that this is caused by a transition of the boundary layer flow over the acrylic glass side plates, since no tripping tape was applied to the side plates. This would lead to a sudden increase in the boundary layer thickness on the side plates and hence a different interaction with the cylinder. A detailed investigation of the effects of side plates on the vortex shedding noise from cylinders, including the coherence between velocity 


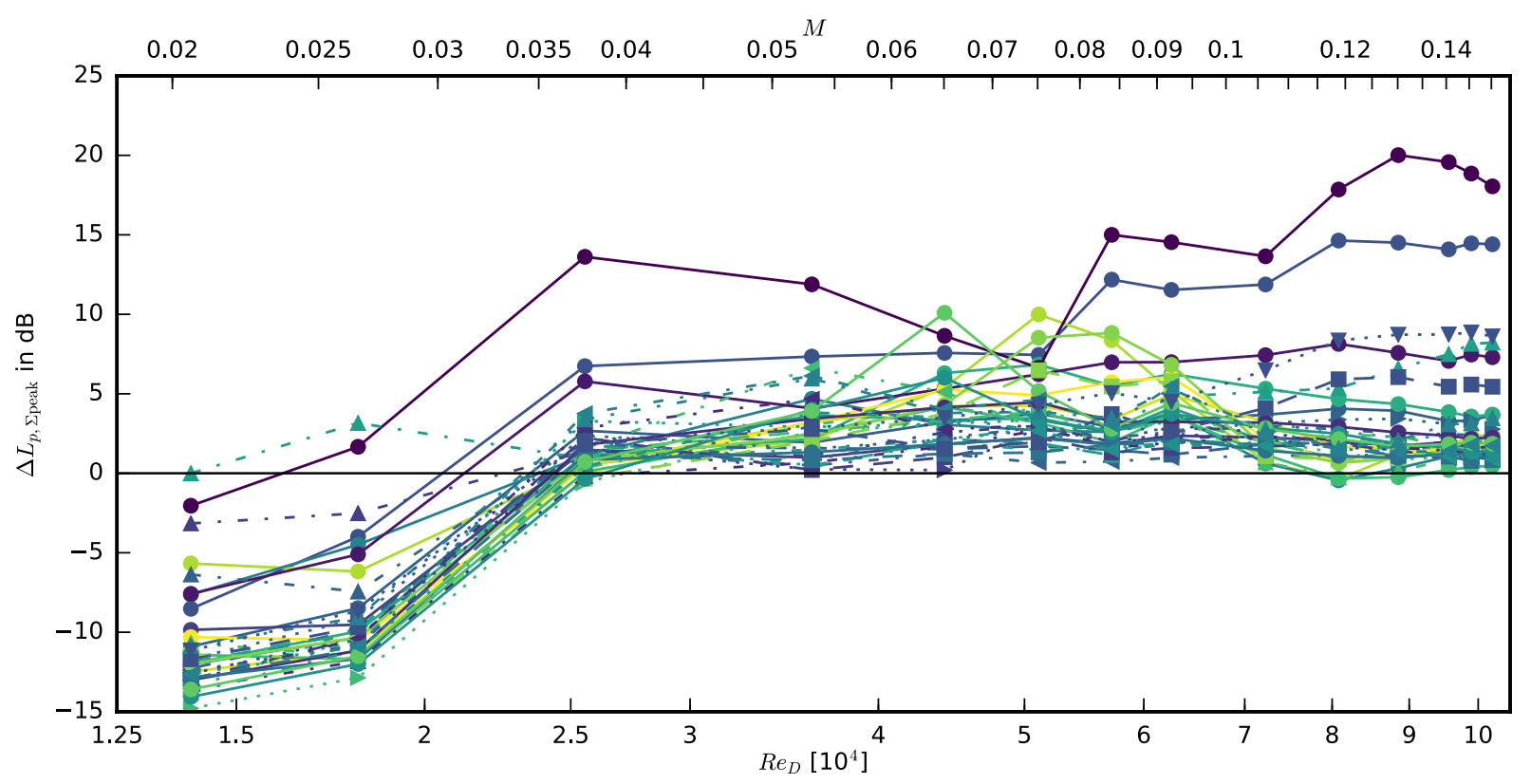

Fig. 9 Difference of the energy in the Kármán vortex shedding peak obtained by the microphone at $90^{\circ}$ for all cylinders from Table 1 , positive values denote noise reduction, negative values denote noise increase, materials are color-coded (porous: $r=1,474,300 \mathrm{~Pa}$ s/ $\mathrm{m}^{2}, \quad 594,200 \mathrm{~Pa} \mathrm{~s} / \mathrm{m}^{2}, \quad 416,200 \mathrm{~Pa} \mathrm{~s} / \mathrm{m}^{2}, \quad 381,500 \mathrm{~Pa} \mathrm{~s} /$ $\mathrm{m}^{2},-314,800 \mathrm{~Pa} \mathrm{~s} / \mathrm{m}^{2},-141,300 \mathrm{~Pa} \mathrm{~s} / \mathrm{m}^{2}$, $112,100 \mathrm{~Pa} \mathrm{~s} /$

fluctuations measured in the cylinder wake and the radiated noise, has been done by Fujita (2010). Another possible cause of the transition-like behavior seen in Fig. 8 is that some change in the boundary layer flow over the cylinders occurs. This needs to be examined in future studies, preferably including more detailed flow measurements such as Particle Image Velocimetry.

Regarding the effect of the porous covers, it can be seen that at Reynolds numbers approximately above 25,000 , the energy obtained for the porous covered cylinders is clearly below that of the reference cylinders, which again confirms the noise reduction potential of such covers. Thereby, the noise reduction obtained by porous materials with low air flow resistivities and high porosities is greater than that obtained for materials with high air flow resistivities and low porosities. For the most effective materials of the present study, the noise reduction at the highest Reynolds numbers exceeds $10 \mathrm{~dB}$. At the two lowest Reynolds numbers of the present study, however, all but one of the porous covers (the one with the lowest air flow resistivity of $4000 \mathrm{~Pa} \mathrm{~s} /$ $\mathrm{m}^{2}$ and a high porosity of $\sigma>0.99$ ) generate a higher peak energy than the non-porous reference cylinders.

In order to be able to evaluate the sound reducing ability of the porous cylinder covers in more detail, the difference of the peak energy will be analyzed. It is calculated according to

$\Delta L_{p, \Sigma \text { peak }}=L_{p, \Sigma \text { peak, reference }}-L_{p, \Sigma \text { peak, porous }}$. $\mathrm{m}^{2}, \quad 86,100 \mathrm{~Pa} \mathrm{~s} / \mathrm{m}^{2}$, 64,500 Pa s $/ \mathrm{m}^{2}$, 53,200 Pa s $/ \mathrm{m}^{2}$, 49,600 Pa s/m², 12,900 Pa s/m², $9800 \mathrm{~Pa} \mathrm{~s} / \mathrm{m}^{2}, 9400 \mathrm{~Pa} \mathrm{~s} /$ $\mathrm{m}^{2}, 4400 \mathrm{~Pa} \mathrm{~s} / \mathrm{m}^{2}, 4100 \mathrm{~Pa} \mathrm{~s} / \mathrm{m}^{2}, 4000 \mathrm{~Pa} \mathrm{~s} / \mathrm{m}^{2}$ ), thicknesses of the porous layer are given by different markers and line styles $(\boldsymbol{\Lambda} d=6 \mathrm{~mm}, \bullet d=10 \mathrm{~mm}, \boldsymbol{\nabla} d=13 \mathrm{~mm}, \boldsymbol{} d=15 \mathrm{~mm}$, $d=20 \mathrm{~mm}, \varangle d=22 \mathrm{~mm}$ )

Thus, a positive value of $\Delta L_{p, \Sigma \text { peak }}$ denotes a reduction in the tonal noise from Kármán vortex shedding due to the porous covers, while a negative value denotes a noise increase. The results are shown in Fig. 9, again as a function of Reynolds number. Basic trends already observed in Fig. 8 can now be seen even more easily. At Reynolds number below 25,000, all porous covers lead to a noise increase. This is especially true for materials with medium to high air flow resistivities, where differences of the peak energy of up to $15 \mathrm{~dB}$ were obtained. For Reynolds numbers above 25,000, all porous covers lead to a notable noise reduction. The only exceptions are porous cylinders covered with Oasis Rainbow Foam $\left(d=10 \mathrm{~mm}, r=416,200 \mathrm{~Pa} \mathrm{~s} / \mathrm{m}^{2}, \sigma=0.97 \ldots 0.98\right)$, Getzner CM GR $052(d=6 \mathrm{~mm}$ and $d=10 \mathrm{~mm}, r=141,300 \mathrm{~Pa} \mathrm{~s} /$ $\left.\mathrm{m}^{2}, \sigma=0.71 \ldots 0.77\right)$ and Conmetall Rubber Mat $(d=10 \mathrm{~mm}$, $r=53,200 \mathrm{~Pa} \mathrm{~s} / \mathrm{m}^{2}, \sigma=0.14 \ldots 0.18$ ), that show a slight noise increase at a Reynolds number around 80,000. Basically, this noise reduction is especially distinctive for materials with low air flow resistivities, where it can take values of up to $20 \mathrm{~dB}$.

As was done for the Strouhal number of the porous covered cylinders, the data shown in Fig. 9 were used to identify an empirical relationship to predict the difference in the energy in the Kármán vortex shedding peak compared to the non-porous reference cylinder, based on the parameters of the porous covers and those of the flow as given in Table 2 . Thereby, the measured peak energies were converted to 
equal those obtained for a cylinder of $1 \mathrm{~m}$ length. Based on the observation that the overall Mach number scaling for the porous covered cylinders is not fundamentally different from that obtained for the non-porous reference cylinder (see Fig. 8), only a dependence on the Reynolds number has been considered. This means that the fundamental scaling with $M^{6}$ observed for Reynolds numbers below 25,000 and above 60,000 as well as the reduced scaling exponent in the range between those two, which may be due to some kind of transition, will be preserved by the model.

Finally, a formulation was chosen from the set of resulting equations that contains all parameters of the porous covers. It was calculated based on 574 data tupels and is given by

$$
\begin{aligned}
\Delta L_{p, \Sigma \text { peak }}= & \left(5.3026+4.1800 \frac{t}{D}+1.7547 \zeta \sigma^{2}\right. \\
& \left.-3.5955 \cdot 10^{9} R e^{-2}-4.6487 \cdot 10^{-5} R e\right) \mathrm{dB} .
\end{aligned}
$$

The prediction model has a goodness of fit of 0.77 and a mean absolute error of $1.6 \mathrm{~dB}$. Again, this equation allows for some basic conclusions on the dependencies of the peak energy difference $\Delta L_{p, \Sigma \text { peak }}$. Firstly, it contains a constant with a positive sign, already revealing that a positive difference, and hence a reduction in the energy in the Kármán vortex shedding peak compared to the reference cylinder due to the porous covers, is likely. Secondly, the energy difference increases with increasing thickness of the porous layer, confirming that, overall, a thick porous layer will lead to a greater noise reduction than a thin porous cover. Thirdly, the third term contains both the normalized permeability $\zeta$ according to Eq. (6) and the porosity $\sigma$. Thus, this summand increases with decreasing air flow resistivity $r$ and increasing porosity, again confirming that a low air flow resistivity and a large porosity are beneficial regarding a high noise reduction. The remaining terms in Eq. (10) contain the dependence on the Reynolds number Re. They lead to a notable increase in the energy difference with increasing $R e$ in the range of low Reynolds numbers and to a very slight decrease in the energy difference with increasing $R e$ at high Reynolds numbers, which agrees well with the overall trend that is visible in Fig. 9. Again, it should be noted that Eq. (10) may not be valid for parameters outside of the ranges specified in Table 2.

The sensitivity of the prediction model given by Eq. (10) was calculated for the input parameters according to Eq. (8), which resulted in a value of 1.79 for the Reynolds number, 0.18 for the normalized permeability $\zeta, 0.13$ for the porosity $\sigma$ and 0.10 for the normalized thickness $t / D$.

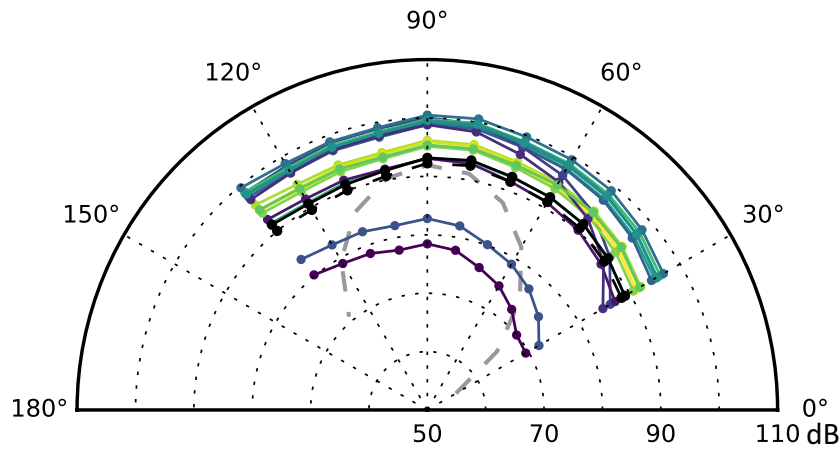

Fig. 10 Directivity of the vortex shedding peak obtained for cylinders covered with different materials at $R e_{D}=99,000, d=10 \mathrm{~mm}$ ( non-porous, $\boldsymbol{n}$ non-porous tripped, porous: $r=\boldsymbol{m}$ 1,474,300 Pa s/ $\mathrm{m}^{2}, \quad 594,200 \mathrm{~Pa} \mathrm{~s} / \mathrm{m}^{2}, \quad 416,200 \mathrm{~Pa} \mathrm{~s} / \mathrm{m}^{2}, 381,500 \mathrm{~Pa} \mathrm{~s} /$ $\mathrm{m}^{2},-314,800 \mathrm{~Pa} \mathrm{~s} / \mathrm{m}^{2},-141,300 \mathrm{~Pa} \mathrm{~s} / \mathrm{m}^{2}, \quad 112,100 \mathrm{~Pa} \mathrm{~s} /$ $\mathrm{m}^{2}, \quad 86,100 \mathrm{~Pa} \mathrm{~s} / \mathrm{m}^{2}, \quad 64,500 \mathrm{~Pa} \mathrm{~s} / \mathrm{m}^{2}, \quad 53,200 \mathrm{~Pa} \mathrm{~s} / \mathrm{m}^{2}$, $49,600 \mathrm{~Pa} \mathrm{~s} / \mathrm{m}^{2}, 12,900 \mathrm{~Pa} \mathrm{~s} / \mathrm{m}^{2},-9800 \mathrm{~Pa} \mathrm{~s} / \mathrm{m}^{2},-9400 \mathrm{~Pa} \mathrm{~s} /$ $\mathrm{m}^{2}, 4400 \mathrm{~Pa} \mathrm{~s} / \mathrm{m}^{2},-4100 \mathrm{~Pa} \mathrm{~s} / \mathrm{m}^{2},-4000 \mathrm{~Pa} \mathrm{~s} / \mathrm{m}^{2},-$ theoretical dipole)

\subsubsection{Tonal noise directivity}

In addition to the analyses of the peak Strouhal number and the peak energy above, the effect of the material properties and the thickness of the porous cover on the directivity of the sound pressure level maximum at the Kármán vortex shedding peak was investigated. This was done using the eleven microphones positioned on one side of the test section at azimuthal angles between $30^{\circ}$ and $130^{\circ}$ (see Fig. 2).

A comparison of the directivity of the vortex shedding noise from the cylinders covered with different porous materials but identical inner diameter $d=0.01 \mathrm{~m}$ is shown in Fig. 10. Basically, it can be concluded that the porous covers for the most part have no effect on the directivity compared to that obtained for the reference cylinder. However, it is also visible that the directivity for two of the cylinders, which are covered with materials with both a low air flow resistivity and a high porosity (Basotect, with $r=9800 \mathrm{~Pa} \mathrm{~s} / \mathrm{m}^{2}, \sigma>0.99$ and Panacell 90 ppi with $r=4000 \mathrm{~Pa} \mathrm{~s} / \mathrm{m}^{2}, \sigma>0.99$ ), shows some differences when compared to the directivity of the nonporous reference cylinder: At small azimuthal angles, approximately between 30 and $80^{\circ}$, and hence in the forward direction, the peak level of the vortex shedding tone is considerably lower than that measured at $90^{\circ}$. A similar effect, although not that distinct, can be seen for the cylinder covered with Packing Foam, the third material characterized by a very low air flow resistivity of $4100 \mathrm{~Pa} \mathrm{~s} / \mathrm{m}^{2}$ and a high porosity $>0.99$. It seems that a low air flow resistivity and a high porosity are not only 
advantageous regarding the overall reduction in the vortex shedding peak and the broadband noise, but they also lead to an additional reduction in the vortex shedding noise in the downstream region. In addition, Fig. 10 contains the theoretical directivity function of a translating dipole,

$D_{r}\left(\theta_{r}\right)=\frac{\sin ^{2} \theta_{r}}{\left(1+M \cos \theta_{r}\right)^{4}}$,

as given by Brooks et al. (1989); Fujita et al. (1999); Hutcheson and Brooks (2012), where $\theta_{r}$ is the angle between the free stream and the observer. It is expressed in the retarded coordinate system (Amiet 1978), which takes into account the refraction at the wind tunnel shear layer on the direction of the emitted noise. As such, it is the angle between the retarded source location and the corrected microphone location (as an example, the ray path from the cylinder to the microphone at a measured angle of $\theta_{m}=60^{\circ}$ and the corresponding corrected microphone location are included in Fig. 2).

It can be seen from Fig. 10 that the measured directivities still differ from that of the theoretical dipole. This may have several reasons, one of which is an additional effect of the shear layer on the amplitude of the sound waves, which is not accounted for in the directivity function given by Eq. (11). This is due to the fact that these corrections are based on very basic models of the shear layer (such as parallel shear layers of constant thickness), which most likely differ from a real shear layer. Another is the fact that the cylinders have a finite length, which means that the microphones will record signals from various spanwise stations, but may potentially also be influenced by some wall-junction effects.

The effect of the thickness of the porous layer on the directivity of the vortex shedding tonal noise is then shown in Fig. 11, again for a material with low air flow resistivity and high porosity (Basotect, $r=9800 \mathrm{~Pa} \mathrm{~s} /$ $\mathrm{m}^{2}, \sigma>0.99$, in Fig. 11a) and for a material with higher air flow resistivity, but lower porosity $\sigma=0.29 \ldots 0.32$ (Damtec SBM K 20, $r=86,100 \mathrm{~Pa} \mathrm{~s} / \mathrm{m}^{2}$, in Fig. 11b). The polar plots show that with increasing thickness of the porous cover, the effect of an additional reduction of the vortex shedding peak in the downstream region can be observed. This effect is stronger for the material with low air flow resistivity (Fig.,11a), but still visible for the material with higher air flow resistivity (Fig. 11b).

\subsection{Flow field measurements}

In this section, results from the CTA measurements will be analyzed and discussed. Thereby, the main aim is to investigate if the hot-wire results can confirm the acoustic

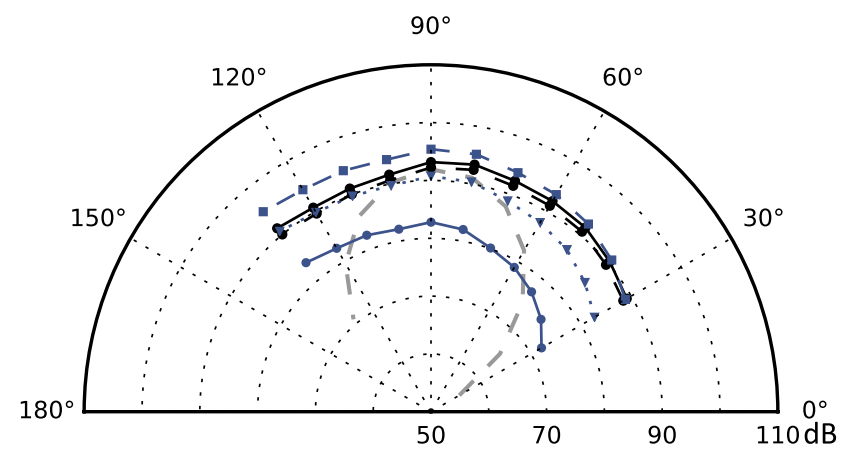

(a) Basotect, $r=9,800 \mathrm{~Pa} \mathrm{~s} / \mathrm{m}^{2}$ ( non-porous, $\mathbf{m}$ nonporous tripped, porous: $\bullet d=10 \mathrm{~mm}, \boldsymbol{\nabla} d=13 \mathrm{~mm}$, $d=20 \mathrm{~mm},-$ theoretical dipole)

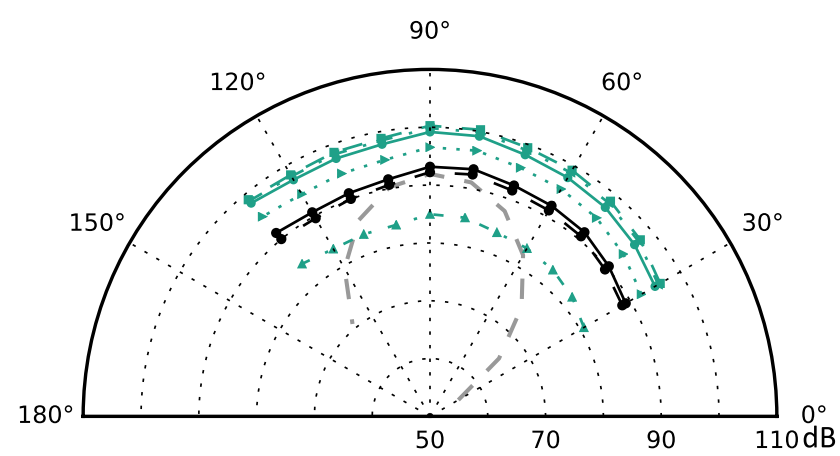

(b) Damtec SBM K 20, $r=86,100 \mathrm{~Pa} \mathrm{~s} / \mathrm{m}^{2}$ ( non-porous, non-porous tripped, porous: $\Delta d=6 \mathrm{~mm}, \bullet d=10 \mathrm{~mm}$, $\checkmark d=15 \mathrm{~mm}, \square d=20 \mathrm{~mm}, \varangle d=22 \mathrm{~mm}$, 므 theoretical dipole)

Fig. 11 Directivity of the vortex shedding peak obtained for cylinders covered with materials of different thickness at $R e_{D}=99,000$

results or if they could even be used to help explaining the acoustic results.

\subsubsection{Wake profiles}

Figures 12 and 13 show profiles of the mean velocity and the turbulence intensity measured in the near wake (one outer diameter downstream from the cylinder axis), obtained for a set of cylinders with different porous materials but identical inner diameter and for a set of cylinders with identical materials, but different thickness of the porous layer, respectively. Due to the symmetry of the problem, only half of the wake profile is shown.

Regarding the influence of the porous materials (Fig. 12) it is visible that, overall, they lead to an increase in the width of the wake, which is in agreement with various past studies (for example Nishimura et al. 1999; Suzuki et al. 2009; Nishimura and Goto 2010; Ashtiani Abdi et al. 2014; Aguiar et al. 2016; Yuan et al. 2016; Xia et al. 2018; Liu et al. 2019; Sadeghipour et al. 2020). Thereby, the width increases with decreasing air flow resistivity and increasing porosity (the 


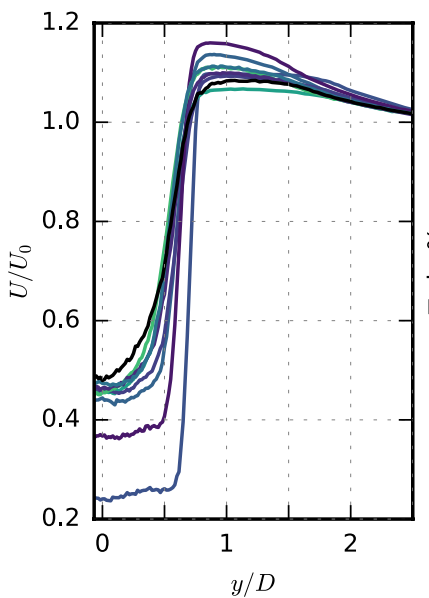

(a) Mean flow velocity

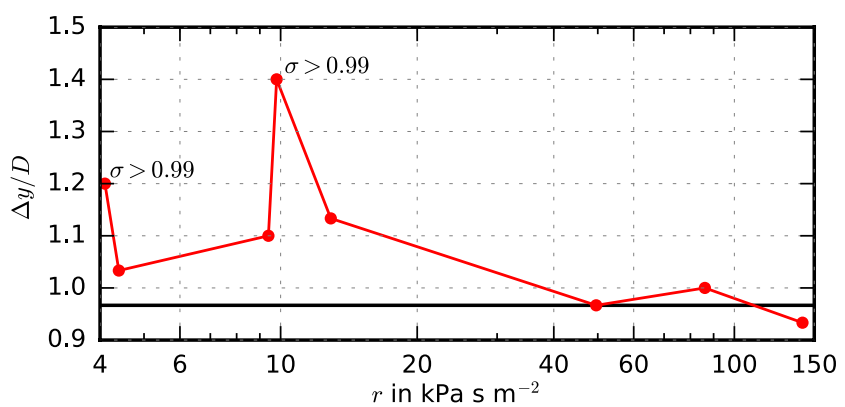

(c) Normalized wake thickness

Fig. 12 Flow characteristics for cylinders covered with different materials at $R e_{D}=70,000$, measured in the near wake $1 \mathrm{D}$ downstream, $d=10 \mathrm{~mm}$ ( non-porous, - non-porous tripped, porous: $r=141,300 \mathrm{~Pa} \mathrm{~s} / \mathrm{m}^{2}, \quad 86,100 \mathrm{~Pa} \mathrm{~s} / \mathrm{m}^{2}, \quad 49,600 \mathrm{~Pa} \mathrm{~s} / \mathrm{m}^{2}$, $12,900 \mathrm{~Pa} \mathrm{~s} / \mathrm{m}^{2}, \quad 9800 \mathrm{~Pa} \mathrm{~s} / \mathrm{m}^{2},-9400 \mathrm{~Pa} \mathrm{~s} / \mathrm{m}^{2}, \quad 4400 \mathrm{~Pa} \mathrm{~s} /$ $\left.\mathrm{m}^{2}, 4100 \mathrm{~Pa} \mathrm{~s} / \mathrm{m}^{2}\right)$

latter will again be discussed below). This can be seen in more detail in Fig. 12(c), where the width of the wake $\Delta y$, normalized with the outer cylinder diameter $D$, is shown as a function of the air flow resistivity $r$ of the porous covers. The width is calculated as the lateral distance between the two points in the velocity profiles where the mean velocity drops below $70 \%$ of the flow speed $U_{0}$. Globally, it can be observed that the width of the wake is greater for materials with low air flow resistivity. However, two local maxima can be seen in the graph, which belong to the two materials that, in addition to a low air flow resistivity, also have a very high porosity $\sigma>0.99$. These are the Packing Foam with $r=4100 \mathrm{~Pa} \mathrm{~s} / \mathrm{m}^{2}$ and Basotect with $r=9800 \mathrm{~Pa} \mathrm{~s} / \mathrm{m}^{2}$. This confirms that a large porosity is also effective in increasing the width of the wake. For the porous material with the highest air flow resistivity of this investigation (Getzner CM GR 0525 with $r=141,3 \mathrm{~Pa} \mathrm{~s} / \mathrm{m}^{2}$ ), the wake is even more slender than for the non-porous reference cylinder.

It can also be observed from Fig. 12a that the mean velocity in the wake of the porous covered cylinders is notably

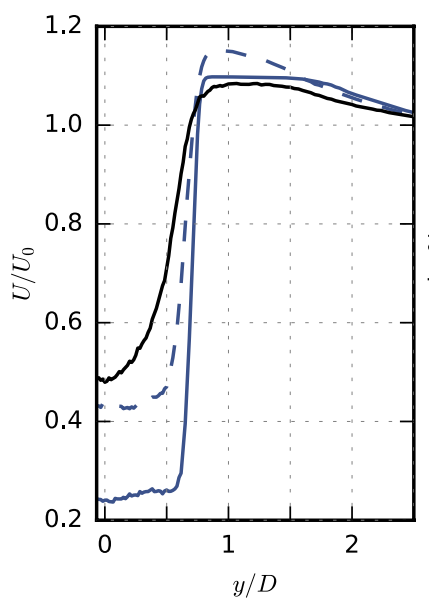

(a) Mean flow velocity

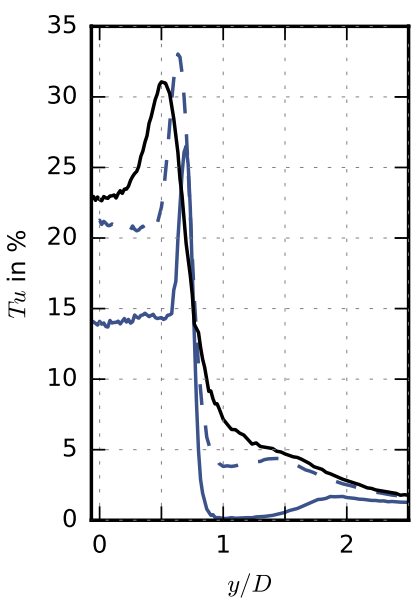

(b) Turbulence intensity

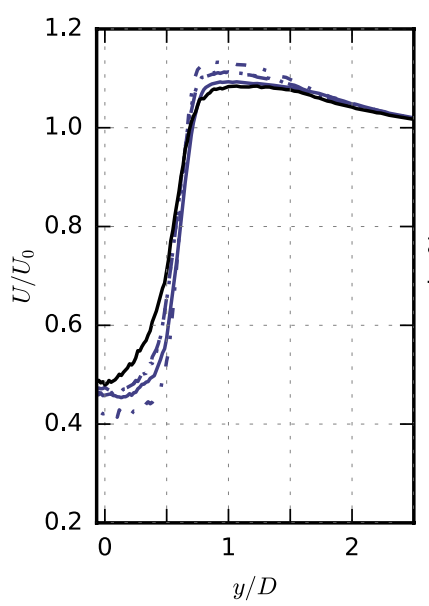

(c) Mean flow velocity

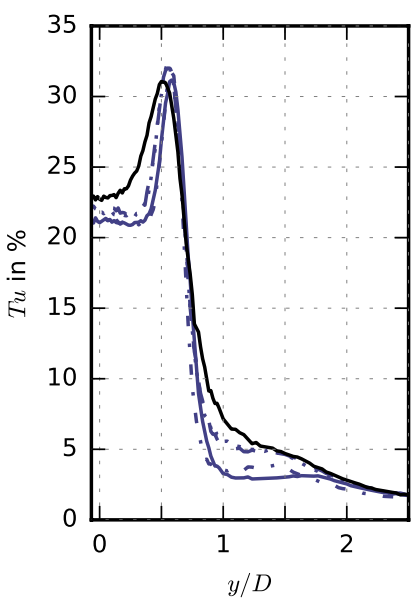

(d) Turbulence intensity

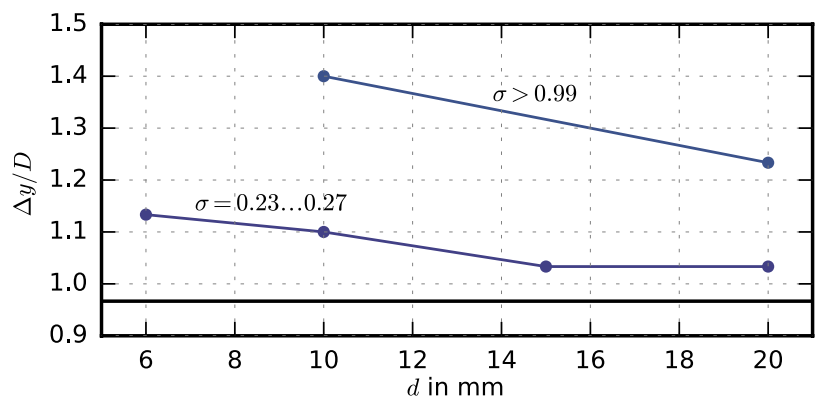

(e) Normalized wake thickness

Fig. 13 Flow characteristics for cylinders covered with materials of different thickness at $R e_{D}=70,000$, measured in the near wake $1 D$ downstream ( non-porous, $\boldsymbol{D}$ non-porous tripped, (a) and (b) porous: $r=9800 \mathrm{~Pa} \mathrm{~s} / \mathrm{m}^{2}, \sigma>0.99, d=10 \mathrm{~mm},=20 \mathrm{~mm}$; (c) and (d) porous: $r=9400 \mathrm{~Pa} \mathrm{~s} / \mathrm{m}^{2}, \sigma=0.23 \ldots 0.27, d=-16 \mathrm{~mm}$, $10 \mathrm{~mm}, \mathrm{~m} 15 \mathrm{~mm}, \mathrm{a} 20 \mathrm{~mm}$; (e) $r=9800 \mathrm{~Pa} \mathrm{~s} / \mathrm{m}^{2}, \sigma>0.99$, $9400 \mathrm{~Pa} \mathrm{~s} / \mathrm{m}^{2}, \sigma=0.23 \ldots 0.27$

reduced compared to the reference cylinder. For the cylinder covered with Basotect ( $r=9800 \mathrm{~Pa} \mathrm{~s} / \mathrm{m}^{2}, \sigma>0.99$ ), which is the one with the smallest wake velocity, it takes a value of approximately $25 \%$ of the outer flow speed $U_{0}$ in 
Fig. 14 Flow characteristics for cylinders covered with different materials at $R e_{D}=70,000$, measured in the far wake $10.5 \mathrm{D}$ downstream, $d=10 \mathrm{~mm}$

( non-porous, $=$ nonporous tripped, porous: $r=141,300 \mathrm{~Pa} \mathrm{~s} / \mathrm{m}^{2}$, $86,100 \mathrm{~Pa} \mathrm{~s} / \mathrm{m}^{2}, \quad 49,600 \mathrm{~Pa} \mathrm{~s} /$ $\mathrm{m}^{2}, 12,900 \mathrm{~Pa} \mathrm{~s} / \mathrm{m}^{2}$, $9800 \mathrm{~Pa} \mathrm{~s} / \mathrm{m}^{2}$, $9400 \mathrm{~Pa} \mathrm{~s} / \mathrm{m}^{2}$, $4400 \mathrm{~Pa} \mathrm{~s} / \mathrm{m}^{2}, 4100 \mathrm{~Pa} \mathrm{~s} /$

$\mathrm{m}^{2}$ )

center of the wake, compared to a value of nearly $50 \%$ for the reference cylinder. Again, this is in good agreement with previous findings (Aguiar et al. 2016; Yuan et al. 2016; Xia et al. 2018; Yuan et al. 2019). In addition to the mean velocity, the porous covers also lead to a reduction of the turbulence intensity in the wake as is visible from Fig. 12b, which agrees with the results from other researchers as well (Suzuki et al. 2009; Nishimura and Goto 2010; Sueki et al. 2010; Gozmen et al. 2013; Aguiar et al. 2016; Showkat Ali et al. 2016; Liu et al. 2019; Sadeghipour et al. 2020). For example, for the cylinder covered with Basotect the turbulence intensity at $y=0$ is about $14 \%$ compared to about $23 \%$ measured for the reference cylinder.

The effect of the thickness of the porous covers on the profiles of mean velocity and turbulence intensity in the near wake is shown in Fig. 13, exemplarily for two different porous materials: Basotect, the melamine foam with a low air flow resistivity of $r=9800 \mathrm{~Pa} \mathrm{~s} / \mathrm{m}^{2}$ and a high porosity $\sigma>0.99$ and Damtec Black Rubber, which consist of a rubber granulate with $r=9400 \mathrm{~Pa} \mathrm{~s} / \mathrm{m}^{2}$ and $\sigma=0.23 \ldots 0.27$. Thus, both materials have a similar air flow resistivity, but a notably different porosity. In agreement with the previous analysis, it can be seen from Fig. 13 that the material with the higher porosity leads to a wider wake as well as to a lower mean velocity and turbulence intensity. Regarding the influence of the thickness of the porous cover, it is visible from Fig. 13a and $\mathrm{c}$ that an increasing thickness (and hence a decreasing inner diameter $d$ ) leads to a reduction of mean velocity and, as shown in Fig. $13 \mathrm{~b}$ and d, to a reduction of turbulence intensity in the wake. The width of the wake reduces with reducing thickness of the cover (Fig. 13e). However, the effect of the thickness is much stronger for the material with high porosity than for the material with low porosity.

The results from the hot-wire measurements performed in the near wake of the porous covered cylinders do not

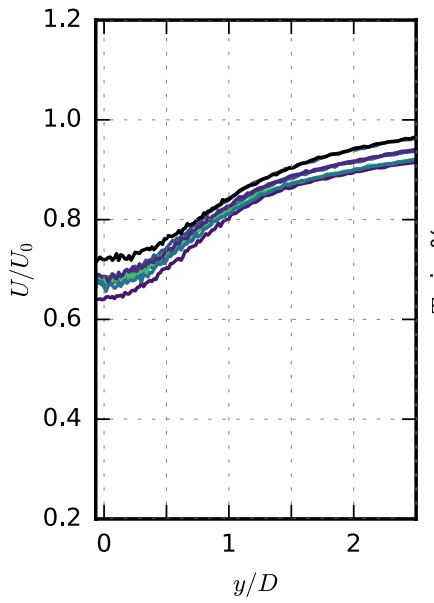

(a) Mean flow velocity

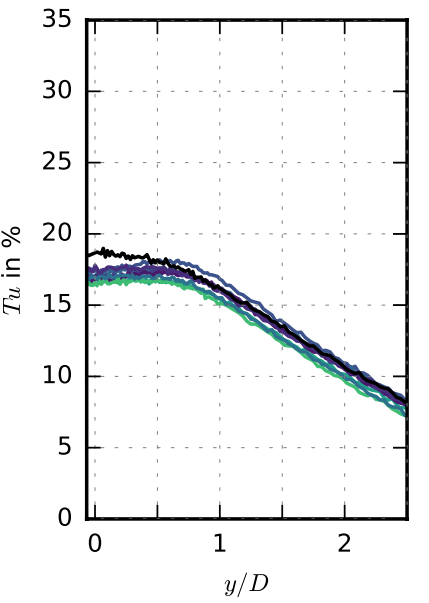

(b) Turbulence intensity show any characteristics that would hint at some kind of vortex shedding from the core cylinder, such as a change of the mean velocity or the turbulence intensity in the region directly downstream of the inner cylinder (between $y=-d / 2$ and $y=+d / 2$ ). Effects like the so-called base bleeding (Naito and Fukagata 2012) cannot be recognized in the results. One possible reason for this observation is that the materials used in the present study are still not permeable enough, meaning that the air flow resistivity is too high. It is reasonable to assume that for cylinder covers with extremely low air flow resistivities (approaching the theoretical value of zero, which would mean that there is virtually no porous material) vortex shedding will occur at the core cylinder, leading to a low-velocity flow through the porous cover and, eventually, to vortex shedding peaks that scale with the inner diameter $d$ rather than with the outer diameter $D$.

The influence of the porous cylinder covers on the profiles of mean velocity and turbulence intensity in the far wake is then shown in Figs. 14 and 15. The measurements were performed at a downstream distance of $10.5 \mathrm{D}$ from the cylinder axis. The influence of the porous material can be observed from Fig. 14, where it is apparent that the porous covers still lead to a reduced mean flow velocity and a reduced turbulence intensity compared to the reference cylinder, although the differences are of course less pronounced than those obtained in the near wake. The effect of the thickness on the far wake is then shown in Fig. 15, where it can be observed that thicker porous covers lead to a further reduced mean velocity and turbulence intensity in the wake. However, this effect is very small only, especially for materials with a low porosity.

Thus, the effects of the porous covers on the profiles of mean velocity and turbulence intensity in the wake are a widening of the wake as well as a notable decrease in the mean velocity and the turbulence intensity. This agrees well with previous studies on porous covered cylinders. 
Fig. 15 Flow characteristics for cylinders covered with materials of different thickness at $R e_{D}=70,000$, measured in the far wake $10 \mathrm{D}$ downstream (non-porous, $\boldsymbol{m}$ non-porous tripped, (a) and (b) porous: $r=9800 \mathrm{~Pa} \mathrm{~s} / \mathrm{m}^{2}, \sigma>0.99$, $d=-10 \mathrm{~mm},=20 \mathrm{~mm}$; (c) and (d) porous: $r=9400 \mathrm{~Pa} \mathrm{~s} /$ $\mathrm{m}^{2}, \sigma=0.23 \ldots 0.27, d=$ $6 \mathrm{~mm}, \quad 10 \mathrm{~mm}, \mathrm{~m}=15 \mathrm{~mm}$, - $20 \mathrm{~mm}$; (e) $r=$ $9800 \mathrm{~Pa} \mathrm{~s} / \mathrm{m}^{2}, \sigma>0.99$, $9400 \mathrm{~Pa} \mathrm{~s} / \mathrm{m}^{2}, \sigma=0.23 \ldots 0.27$

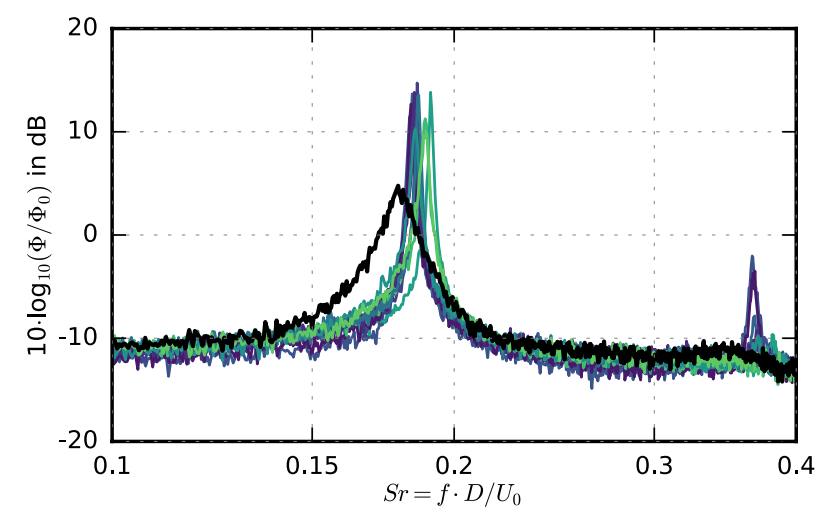

Fig. 16 Spectra of the turbulent velocity fluctuations obtained for cylinders covered with different porous materials of the same thickness $\left(d=10 \mathrm{~mm},-\right.$ non-porous, porous: $r=314,800 \mathrm{~Pa} \mathrm{~s} / \mathrm{m}^{2}$, - 141,300 Pa s/m², 86,100 Pa s/m², 64,500 Pa s/m², 49,600 Pa s/m², 12,900 Pa s/m², $9800 \mathrm{~Pa} \mathrm{~s} / \mathrm{m}^{2}, 9400 \mathrm{~Pa} \mathrm{~s} /$ $\mathrm{m}^{2}, 4400 \mathrm{~Pa} \mathrm{~s} / \mathrm{m}^{2}, 4100 \mathrm{~Pa} \mathrm{~s} / \mathrm{m}^{2}$ ) at $R e_{D}=102,600$ using CTA

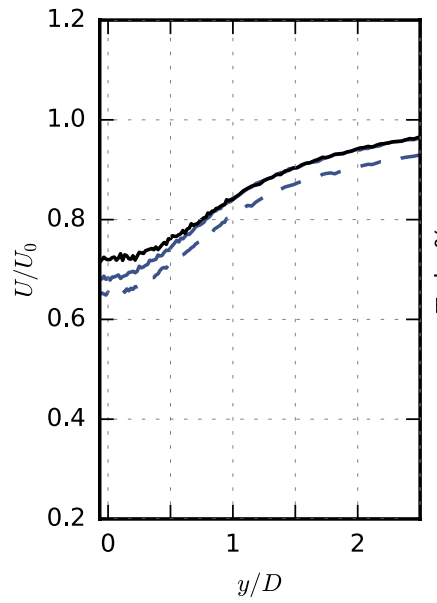

(a) Mean flow velocity

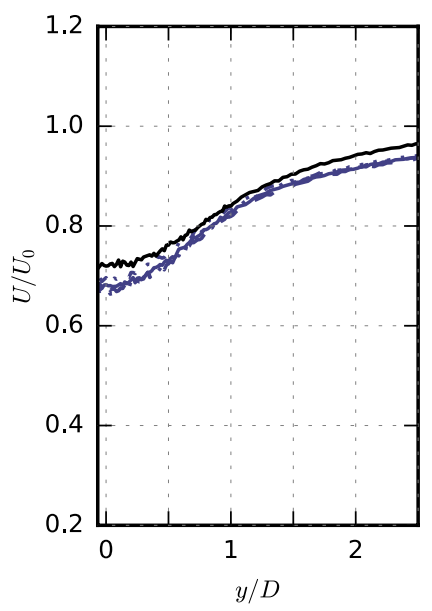

(c) Mean flow velocity

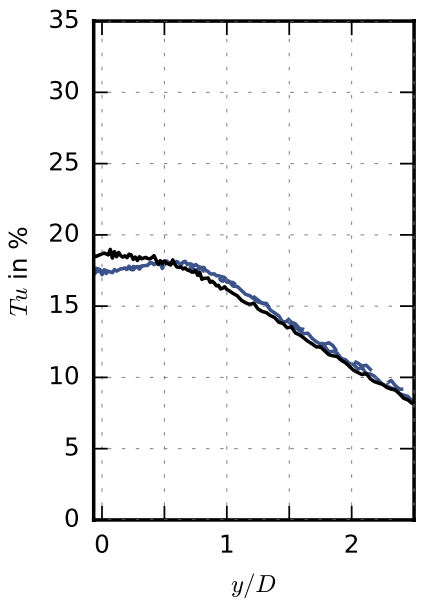

(b) Turbulence intensity

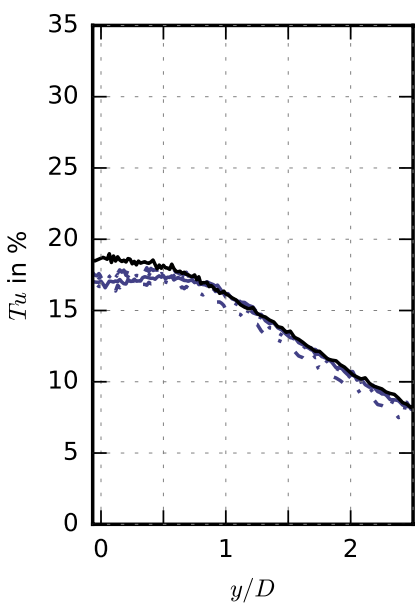

(d) Turbulence intensity

\subsubsection{Turbulence spectral densities}

Similar to the presentation of the acoustic results, spectra of the turbulent velocity fluctuations measured in the wake of selected porous covered cylinders are shown exemplarily in Figs. 16 and 17. This includes a comparison of turbulence spectra obtained for cylinders with the same inner diameter of $d=10 \mathrm{~mm}$, covered with different porous materials (Fig. 16) as well as a comparison of spectra obtained for cylinders covered with the same material, but different thicknesses. This was done for two materials, Basotect, the melamine foam with an air flow resistivity of $9800 \mathrm{~Pa} s / \mathrm{m}^{2}$ and a porosity $>0.99$ (Fig. 17a) and Damtec Black Rubber, a rubber granulate with a similar air flow resistivity of $9400 \mathrm{~Pa} \mathrm{~s} / \mathrm{m}^{2}$, but a notably lower porosity between 0.23 and 0.27 (Fig. 17b). In general, it is immediately visible that the porous covers lead to a narrower vortex shedding peak, but a higher peak level, compared to the non-porous reference cylinder. Basically, this is in agreement with the results from the acoustic measurements presented in Sect. 3.1, however, the peak amplitude of the velocity fluctuations of the 


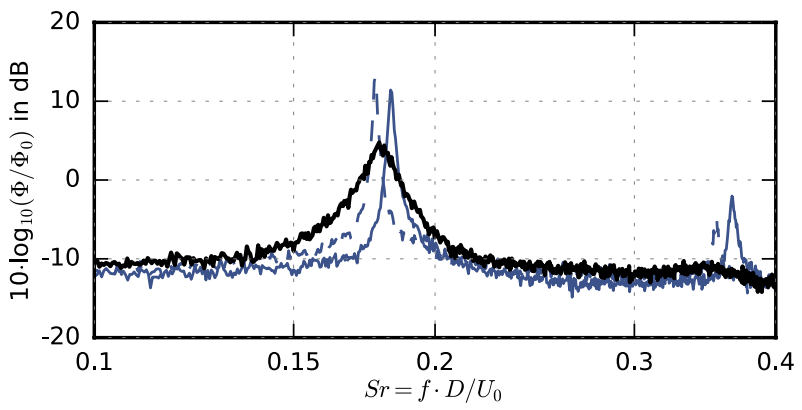

(a) Basotect, $r=9,800 \mathrm{~Pa} \mathrm{~s} / \mathrm{m}^{2}, \sigma>0.99$, non-porous, porous: $d=10 \mathrm{~mm}$, $\boldsymbol{E} d=20 \mathrm{~mm}$

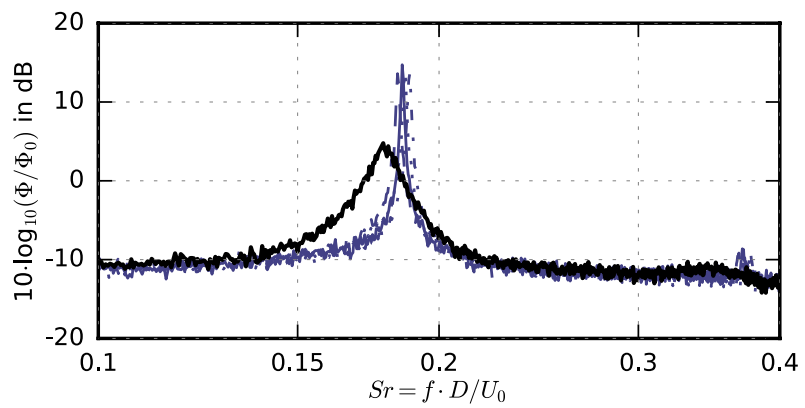

(b) Damtec Black Rubber, $r=9,400 \mathrm{~Pa} \mathrm{~s} / \mathrm{m}^{2}$, $\sigma=0.23 \ldots 0.27,-$ non-porous, porous: $-d=6 \mathrm{~mm}$, $d=10 \mathrm{~mm}, \boldsymbol{\square} d=15 \mathrm{~mm}$, 마 $d=20 \mathrm{~mm}$

Fig. 17 Spectra of the turbulent velocity fluctuations obtained for cylinders covered with porous materials of different thickness at $R e_{D}=102,600$ using CTA porous covered cylinders exceeds that from the reference cylinder far more than that was the case for the corresponding peak amplitudes of the sound pressure levels as shown in Figs. 4 and 5. Thus, the hot-wire data confirm the results from the acoustic measurements, suggesting that the same mechanisms are responsible both for the modification of the flow field and for the noise radiated to the far field. Another interesting effect that can be observed for materials with low air flow resistivities (especially visible in Fig. 16) is that a second maximum appears in the turbulence spectra, at twice the Strouhal number of the vortex shedding peak, which does not appear in the spectra obtained for the reference cylinder. This is the first harmonic of the vortex shedding tone, which is known to be related to unsteady drag induced on the cylinders (Casalino and Jacob 2003). Analyzing the results obtained for the cylinders covered with the melamine foam of different thicknesses, as shown in Fig. 17a, reveals a strong influence of the material thickness. Here, the vortex shedding peak of the cylinder with the thinner cover $(d=20 \mathrm{~mm})$ appears at a Strouhal number which is slightly smaller than that of the reference cylinder, while the cylinder covered with the thicker porous layer $(d=10 \mathrm{~mm})$ generates a peak at a slightly higher Strouhal number. Such an effect cannot be observed for the porous rubber, which has a considerably lower porosity, as shown in Fig. 17b. There, the peaks of the porous covered cylinders always lead to a Strouhal number slightly above that of the reference cylinder. This again emphasizes that not only the air flow resistivity, and

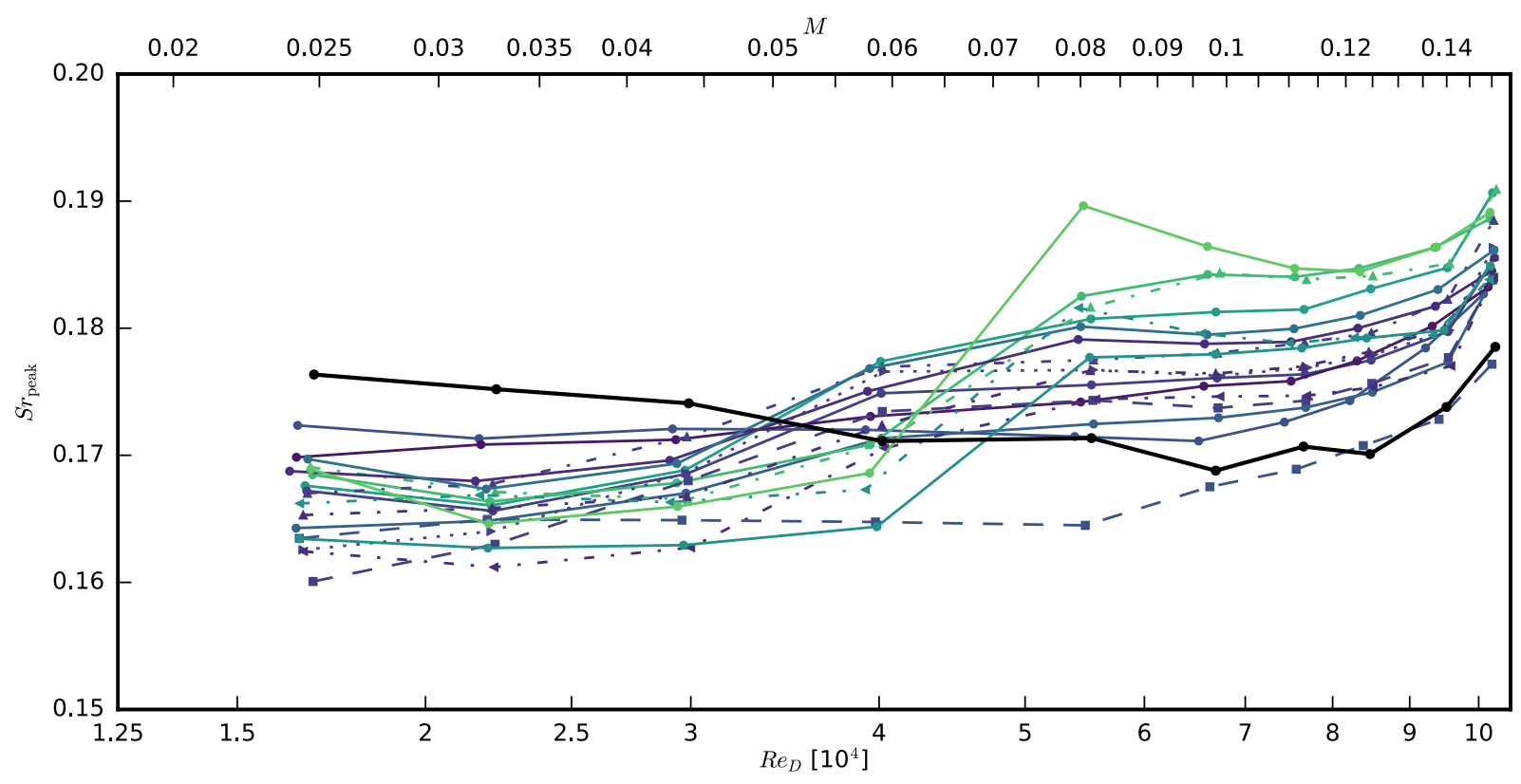

Fig. 18 Strouhal number of the Kármán vortex shedding peak for all cylinders from Table 1 obtained from CTA measurements, materials are color-coded ( non-porous, porous: $r=-314,800 \mathrm{~Pa}$ s/ $\mathrm{m}^{2}, 141,300 \mathrm{~Pa} \mathrm{~s} / \mathrm{m}^{2}, 86,100 \mathrm{~Pa} \mathrm{~s} / \mathrm{m}^{2}, \quad 64,500 \mathrm{~Pa} \mathrm{~s} / \mathrm{m}^{2}$,
49,600 Pa s $/ \mathrm{m}^{2}, \quad 12,900 \mathrm{~Pa} \mathrm{~s} / \mathrm{m}^{2}, 9800 \mathrm{~Pa} \mathrm{~s} / \mathrm{m}^{2}, 9400 \mathrm{~Pa} \mathrm{~s} /$ $\mathrm{m}^{2}$, $4400 \mathrm{~Pa} \mathrm{~s} / \mathrm{m}^{2},-4100 \mathrm{~Pa} \mathrm{~s} / \mathrm{m}^{2}$ ), thicknesses of the porous layer are given by different markers and line styles $(\Delta d=6 \mathrm{~mm}$,

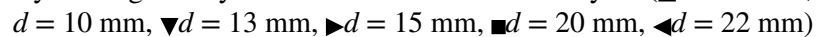




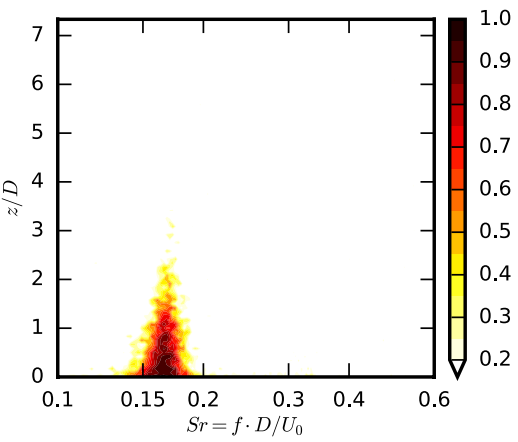

(a) Reference

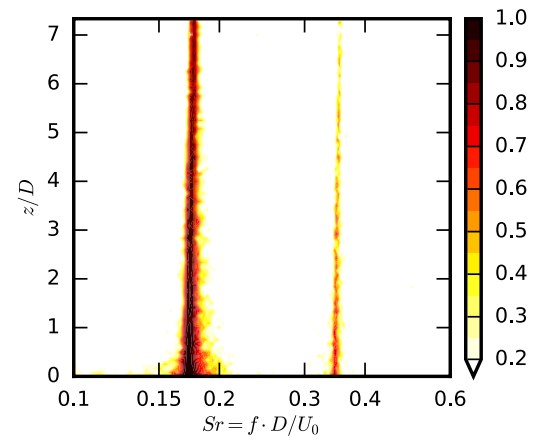

(d) $r=9,800 \mathrm{~Pa} \mathrm{~s} / \mathrm{m}^{2}, d=10 \mathrm{~mm}$

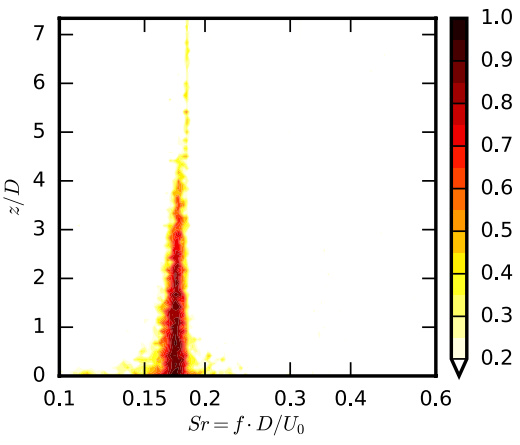

(b) $r=141,300 \mathrm{~Pa} \mathrm{~s} / \mathrm{m}^{2}, d=10 \mathrm{~mm}$

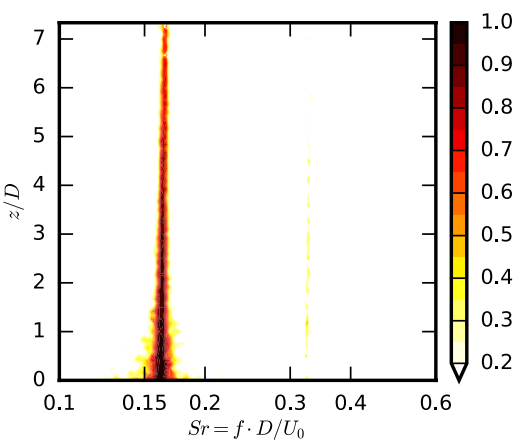

(e) $r=9,800 \mathrm{~Pa} \mathrm{~s} / \mathrm{m}^{2}, d=20 \mathrm{~mm}$

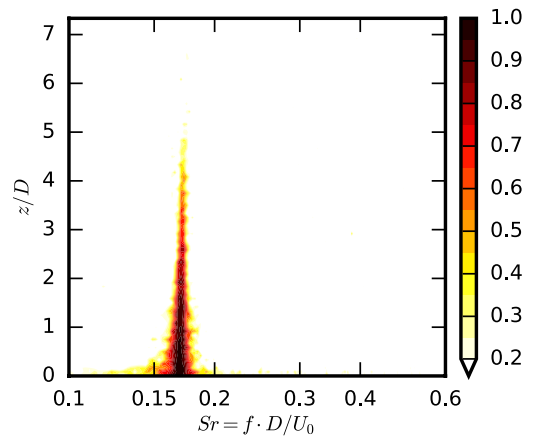

(c) $r=64,500 \mathrm{~Pa} \mathrm{~s} / \mathrm{m}^{2}, d=10 \mathrm{~mm}$

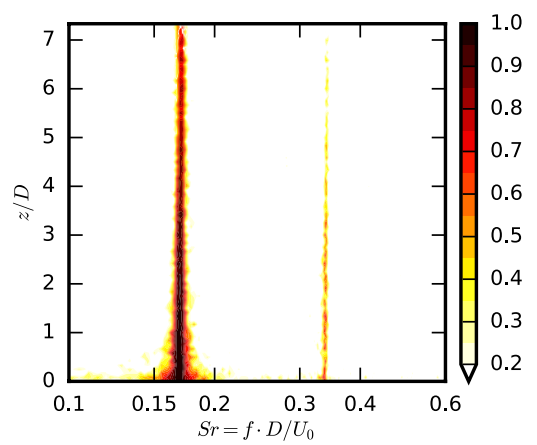

(f) $r=4,100 \mathrm{~Pa} \mathrm{~s} / \mathrm{m}^{2}, d=10 \mathrm{~mm}$

Fig. 19 Spanwise coherence $\gamma^{2}$, calculated according to Eq. (3) from measurements performed downstream of the cylinders at $R e_{D}=77,000$

thus the permeability of the porous material, is important, but also its porosity.

In agreement with the analyses of the acoustic results and the wake measurements above, the spectra of the turbulent velocity fluctuations do not show any characteristics that could be related to a shedding of vortices from the core cylinder.

It is also visible from Figs. 16 and 17 that the porous covers have an influence on the broadband content of the velocity spectra, which again agrees with the results from the acoustic measurements. However, due to the fact that these measurements were conducted at a fixed location with respect to the cylinder position, differences in the location of the shear layers due to the widening of the wake observed for the porous materials were not taken into account. Thus, the amplitudes of the velocity spectra should be interpreted with some care.

Similar to the analysis of the acoustic results as presented in Sect. 3.1, the spectral peaks of the velocity fluctuations obtained by the CTA measurements will be analyzed regarding their Strouhal number. Due to the aforementioned fact that the measurements did not take into account the variations of the exact shear layer position, the peak level and the energy in the peak of the velocity spectra are not evaluated. Accordingly, Fig. 18 shows the vortex shedding Strouhal number, obtained from the
CTA measurements, as a function of the Reynolds number $R e_{D}$. When compared with the Strouhal numbers obtained from the sound pressure level spectra as shown in Fig. 7, the same general behavior is visible: At Reynolds numbers approximately below 35,000 , the peak Strouhal number obtained for the porous covered cylinders is below that obtained for the non-porous reference cylinder. With increasing Reynolds number, the Strouhal number for the reference cylinder slightly decreases, while that for the porous covered cylinders gradually increases. Thus, at Reynolds numbers roughly above 40,000 the Strouhal number is greater for the porous covered cylinders. Regarding the influence of the material parameters, it can again be seen that porous materials with high air flow resistivities lead to higher Strouhal numbers than materials with low air flow resistivities. The fact that the Strouhal numbers obtained from the hot-wire measurements in general are lower than those obtained from the acoustic measurements is mainly caused by the additional blockage due to the traverse system.

In general, the analysis of the vortex shedding peak of the velocity fluctuations in the wake of the cylinders basically revealed similar trends as the analysis of the vortex shedding peak in the far field sound pressure level spectra. As mentioned above, this shows that the porous covers exert the same effect on the velocity fluctuations in the adjacent 
flow field as on the pressure fluctuations radiated as noise. One potential mechanism of the porous covers is hydrodynamic absorption. This term describes the viscous damping of turbulent flow pressure amplitudes (Herr 2007), meaning that the porous materials absorb energy from turbulent eddies that flow over and interact with the porous surface. The present results support this thesis, since the spectra of the turbulence in the shear layer show the same narrowing of the vortex shedding peak as the acoustic spectra. Further supporting this theory is the fact that in the current study, the best results were obtained for those materials that have a low air flow resistivity and a high porosity, which are usually characterized by rather large open pores. Large pores, of course, enable a better absorption of turbulent eddies than small pores. In addition, hydrodynamic absorption would also account for the reduction in broadband noise discussed in Sect. 3.1.2, since the analysis of velocity spectral densities showed a reduction in the broadband content as well.

\subsubsection{Spanwise coherence}

Finally, in order to allow for more insight into the potential noise reduction mechanism of the porous covers, the spanwise coherence was measured and calculated according to Eq. (3). Due to the fact that these measurements are very time consuming, this was only done for a small subset of the porous covered cylinders. The results are shown in Fig. 19. It is clearly visible that the coherence measured for the non-porous reference cylinder has a notably smaller spanwise extent than that measured for the cylinders with porous cover. This is a really interesting result, since it signifies that the noise reduction observed for porous covered cylinders is not caused by a disturbance of spanwise coherent structures. The coherence measured for the porous covered cylinders shows similar features like the spectra of both the acoustic measurements and the hot-wire measurements, mainly a notably narrower peak. Basically, this means that the turbulent velocity fluctuations in the shear layer of the porous covered cylinders not only have a higher peak level (as shown in Figs. 16 and 17), but they are also correlated along a greater extent of the span. Most, but not all, of the maximum far field sound pressure levels measured for the porous covered cylinders (see Figs. 4 and 5) also exceed the level measured for the reference cylinder, although the differences are not so large. This implies that additional effects may be important, such as damping or nonlinear effects when the noise travels to the far field. In addition, it is also visible from Fig. 19 that peaks of the coherence at twice the vortex shedding frequency appear for cylinder covers with low air flow resistivity. This was also observed in the velocity spectra of the porous covered cylinders.

\section{Summary}

An extensive wind tunnel study was performed in order to investigate the effect of porous covers on the aerodynamic noise generated by circular cylinders in a subsonic flow. To this end, a total of 17 different materials were used to cut porous covers of different thickness. The cylinders all had a length of $0.28 \mathrm{~m}$ and an outer diameter of $0.03 \mathrm{~m}$, resulting in an aspect ratio of 9.3. For means of comparison, a nonporous reference cylinder with the same length and (outer) diameter was used as a baseline. Experiments were performed in an open jet aeroacoustic wind tunnel at Reynolds numbers approximately ranging from 14,000 to 103,000 . In addition to the acoustic measurements, which were done using a set of far field microphones, CTA measurements were performed to allow for conclusions on the effect of the porous covers on the flow field.

In agreement with previous studies (Liu et al. 2012; Geyer and Sarradj 2016; Klausmann and Ruck 2017), the acoustic measurements showed that the porous covers used in this study do not lead to a complete suppression of the vortex shedding tone, but to a considerable narrowing of the tonal peak. It can be assumed that this is due to the fact that the materials were limited to those with rather medium and high air flow resistivities. As was found by several other researchers, materials with a higher permeability will much likely reduce the vortex shedding noise completely (see, for example, Nishimura et al. 1999; Sueki et al. 2010; Nishimura and Goto 2010; Aguiar et al. 2016). Overall, of course, the narrowing of the vortex shedding tone observed in the present work will still lead to a reduction in the aerodynamic noise caused by the porous covers. Due to the large amount of experimental data, basic trends for the estimation of key parameters of the vortex shedding tonal noise were derived using the method of linear regression. These parameters include the Strouhal number of the vortex shedding peak and the reduction of energy in the Kármán vortex shedding peak due to the porous covers. For the peak Strouhal number, it was found that increasing the air flow resistivity and the thickness as well as decreasing the porosity of the porous materials will lead to an increase in the Strouhal number. Likewise, an increase in the porosity and the thickness as well as a decrease in the air flow resistivity will also lead to an increase in the reduction of peak energy. In addition to the reduction of tonal noise, the porous cylinder covers also lead to a notable reduction of broadband noise, where again materials with low air flow resistivity and high porosity are most efficient.

CTA measurements in the wake showed an increase in the wake width for the porous covered cylinders compared to the reference cylinder as well as a decrease in mean velocity 
and turbulence intensity in the wake, which agrees well with results from other researchers. The analysis of spectra of the turbulent velocity fluctuations in the wake of a subset of the porous covered cylinders generally revealed similar behavior as that observed for the sound pressure level spectra: The spectral peak due to the Kármán vortex shedding is much narrower, albeit having a higher peak level compared to the reference cylinder. Additional measurements showed that the spanwise coherence obtained for the porous covered cylinders is notably longer in the spanwise direction than that of the reference cylinder. Thus, the underlying mechanism of the noise reduction is not a disturbance of the spanwise coherence. It is rather likely that hydrodynamic absorption plays an important role, since the porous covers seem to have the same effect on the turbulence in the adjacent flow (the shear layers) as on the far field noise.

Acknowledgements Open Access funding provided by Projekt DEAL. The author thanks Julia Genßler and Richard Peschel for their help with the measurements and Ennes Sarradj for the guidance and the helpful discussions.

\section{Compliance with ethical standards}

Conflict of interest The author declares that he has no conflict of interest.

Open Access This article is licensed under a Creative Commons Attribution 4.0 International License, which permits use, sharing, adaptation, distribution and reproduction in any medium or format, as long as you give appropriate credit to the original author(s) and the source, provide a link to the Creative Commons licence, and indicate if changes were made. The images or other third party material in this article are included in the article's Creative Commons licence, unless indicated otherwise in a credit line to the material. If material is not included in the article's Creative Commons licence and your intended use is not permitted by statutory regulation or exceeds the permitted use, you will need to obtain permission directly from the copyright holder. To view a copy of this licence, visit http://creativecommons.org/licenses/by/4.0/.

\section{References}

Aguiar J, Yao H, Liu Y (2016) Passive flow/noise control of a cylinder using metal foam. In: Proceedings of the 23rd international congress on sound and vibration, pp 1-8

Akishita S, Yahathugoda I (2005) Effect of surface impedance for reducing aerodynamic sound from circular cylinder. In: 11th AIAA/CEAS aeroacoustics conference, p 2914

Amiet R (1978) Refraction of sound by a shear layer. J Sound Vib 58(4):467-482

Arcondoulis EJ, Liu Y, Li Z, Yang Y, Wang Y (2019) Structured porous material design for passive flow and noise control of cylinders in uniform flow. Materials 12(18):2905

Ashtiani Abdi I, Hooman K, Khashehchi M (2014) A comparison between the separated flow structures near the wake of a bare and a foam-covered circular cylinder. J Fluids Eng 136(12)

Bearman PW (1969) On vortex shedding from a circular cylinder in the critical Reynolds number regime. J Fluid Mech 37(3):577-585
Bendat JS, Piersol AG (2010) Random data: analysis and measurement procedures, 4th, edition edn. Wiley, Amsterdam

Bhattacharyya S, Singh A (2011) Reduction in drag and vortex shedding frequency through porous sheath around a circular cylinder. Int J Numer Methods Fluids 65(6):683-698

Blake W.K (ed) (1986) Mechanics of flow-induced sound and vibration volume 1: general concepts and elementary sources, chap. Dipole Sound from Cylinders. Academic Press Inc., London

Brooks TF, Pope DS, Marcolini MA (1989) Airfoil self-noise and prediction. Tech. rep., NASA Reference Publication 1218

Bruneau CH, Mortazavi I (2008) Numerical modelling and passive flow control using porous media. Comput Fluids 37(5):488-498

Casalino D, Jacob M (2003) Prediction of aerodynamic sound from circular rods via spanwise statistical modelling. J Sound Vib 262(4):815-844

Fey U, König M, Eckelmann H (1998) A new Strouhal-Reynolds-number relationship for the circular cylinder in the range $47<\operatorname{Re}<2 x$ 10 5. Phys Fluids 10(7):1547-1549

Fujita $H$ (2010) The characteristics of the aeolian tone radiated from two-dimensional cylinders. Fluid Dyn Res 42(1):015002

Fujita H, Suzuki H, Sagawa A, Takaishi T (1999) The aeolian tone characteristics of a circular cylinder in high Reynolds number flow. In: 5th AIAA/CEAS aeroacoustics conference and exhibit, pp AIAA paper 1999-1849

Geyer TF (2011) Trailing edge noise generation of porous airfoils. $\mathrm{Ph} . \mathrm{D}$. thesis, Brandenburg University of Technology, Cottbus

Geyer TF (2020) Vortex shedding noise from finite, wall-mounted, circular cylinders modified with porous material. AIAA J 58(5):1-15

Geyer TF, Sarradj E (2016) Circular cylinders with soft porous cover for flow noise reduction. Exp Fluids 57(3):30

Geyer TF, Sarradj E (2019) Self noise reduction and aerodynamics of airfoils with porous trailing edges. Acoustics 1(2):393-409

Geyer TF, Sarradj E, Fritzsche C (2010) Measurement of the noise generation at the trailing edge of porous airfoils. Exp Fluids 48(2):291-308

Gozmen B, Firat E, Akilli H, Sahin B (2013) Flow control behind a circular cylinder via a porous cylinder in deep water. In: EPJ web of conferences, vol 45, p 01035. EDP Sciences

Herr M (2007) Design criteria for low-noise trailing-edges. In: 13th AIAA/CEAS aeroacoustics conference), pp AIAA paper 2007-3470

Hutcheson FV, Brooks TF (2012) Noise radiation from single and multiple rod configurations. Int J Aeroacoust 11(3-4):291-333

Igarashi T (1986) Effect of tripping wires on the flow around a circular cylinder normal to an airstream. Bull JSME 29(255):2917-2924

ISO 9053 (1993) Acoustics - materials for acoustical applicationsdetermination of airflow resistance. Tech. rep., International Organization for Standardization

Kamps L, Geyer TF, Sarradj E, Brücker C (2017) Vortex shedding noise of a cylinder with hairy flaps. J Sound Vib 388:69-84

Keefe RT (1962) Investigation of the fluctuating forces acting on a stationary circular cylinder in a subsonic stream and of the associated sound field. J Acoust Soc Am 34(11):1711-1714

Klausmann K, Ruck B (2017) Drag reduction of circular cylinders by porous coating on the leeward side. J Fluid Mech 813:382-411

Liu F, Guo H, Hu T, Liu P (2019) Experimental investigation on the aeroacoustics of circular cylinders covered with metal foam. In: 25th AIAA/CEAS aeroacoustics conference, pp AIAA paper 2019-2715

Liu H, Wei J, Qu Z (2012) Prediction of aerodynamic noise reduction by using open-cell metal foam. J Sound Vib 331(7):1483-1497

Moreau DJ, Geyer TF, Doolan CJ, Sarradj E (2018) Surface curvature effects on the tonal noise of a wall-mounted finite airfoil. J Acoust Soc Am 143(6):3460-3473

Naito H, Fukagata K (2012) Numerical simulation of flow around a circular cylinder having porous surface. Phys Fluids 24(11):117102 
Nishimura M, Goto T (2010) Aerodynamic noise reduction by pile fabrics. Fluid Dyn Res 42(1):015003

Nishimura M, Kudo T, Nishioka M (1999) Aerodynamic noise reducing techniques by using pile-fabrics. In: 5th AIAA/CEAS aeroacoustics conference and exhibit, pp AIAA paper 1999-1847

Porteous R, Moreau DJ, Doolan CJ (2014) A review of flow-induced noise from finite wall-mounted cylinders. J Fluids Struct $51: 240-254$

Rashidi S, Hayatdavoodi M, Esfahani JA (2016) Vortex shedding suppression and wake control: a review. Ocean Eng 126:57-80

Rashidi S, Tamayol A, Valipour MS, Shokri N (2013) Fluid flow and forced convection heat transfer around a solid cylinder wrapped with a porous ring. Int J Heat Mass Transf 63:91-100

Sadeghipour S, Ali SAS, Liu X, Azarpeyvand M, Thorpe GR (2020) Control of flows around bluff bodies mediated by porous materials. Exp Therm Fluid Sci 114:110048

Sarradj E, Fritzsche C, Geyer TF, Giesler J (2009) Acoustic and aerodynamic design and characterization of a small-scale aeroacoustic wind tunnel. Appl Acoust 70(8):1073-1080

Sarradj E, Geyer TF (2014) Symbolic regression modeling of noise generation at porous airfoils. J Sound Vib 333(14):3189-3202

Schmidt M, Lipson H (2009) Distilling free-form natural laws from experimental data. Science 324(5923):81-85

Showkat Ali S.A, Liu X, Azarpeyvand M (2016) Bluff body flow and noise control using porous media. In: 22nd AIAA/CEAS aeroacoustics conference, p 2754

Strouhal V (1878) Über eine besondere Art der Tonerregung. Ann Phys 241(10):216-251

Sueki T, Takaishi T, Ikeda M, Arai N (2010) Application of porous material to reduce aerodynamic sound from bluff bodies. Fluid Dyn Res 42(1):015004
Suzuki M, Sueki T, Takaishi T, Nakade K (2009) A numerical study on mechanism of aerodynamic noise reduction by porous material. In: The sixteenth international congress on sound and vibration, ICSV, vol 16

Willis MJ, Hiden HG, Marenbach P, McKay B, Montague GA (1997) Genetic programming: an introduction and survey of applications. In: Second international conference on genetic algorithms in engineering systems: innovations and applications, pp 314-319. IET

Xia C, Wei Z, Yuan H, Li Q, Yang Z (2018) Pod analysis of the wake behind a circular cylinder coated with porous media. J Vis 21(6):965-985

Xu Y, Zheng ZC, Wilson D (2011) A computational study of the effect of windscreen shape and flow resistivity on turbulent wind noise reduction. J Acoust Soc Am 129(4):1740-1747

Yuan H, Xia C, Chen Y, Yang Z (2016) Flow around a finite circular cylinder coated with porous media. In: Proceedings of the 8th international colloquium on bluff body aerodynamics and applications

Yuan Y, Xu K, Zhao K (2019) Numerical analysis of transport in porous media to reduce aerodynamic noise past a circular cylinder by application of porous foam. J Therm Anal Calorim 1-12

Zhao M, Cheng L (2010) Finite element analysis of flow control using porous media. Ocean Eng 37(14-15):1357-1366

Zhao S, Dabin M, Cheng E, Qiu X, Burnett I, Liu JCc (2017) On the wind noise reduction mechanism of porous microphone windscreens. J Acoust Soc Am 142(4):2454-2463

Publisher's Note Springer Nature remains neutral with regard to jurisdictional claims in published maps and institutional affiliations. 